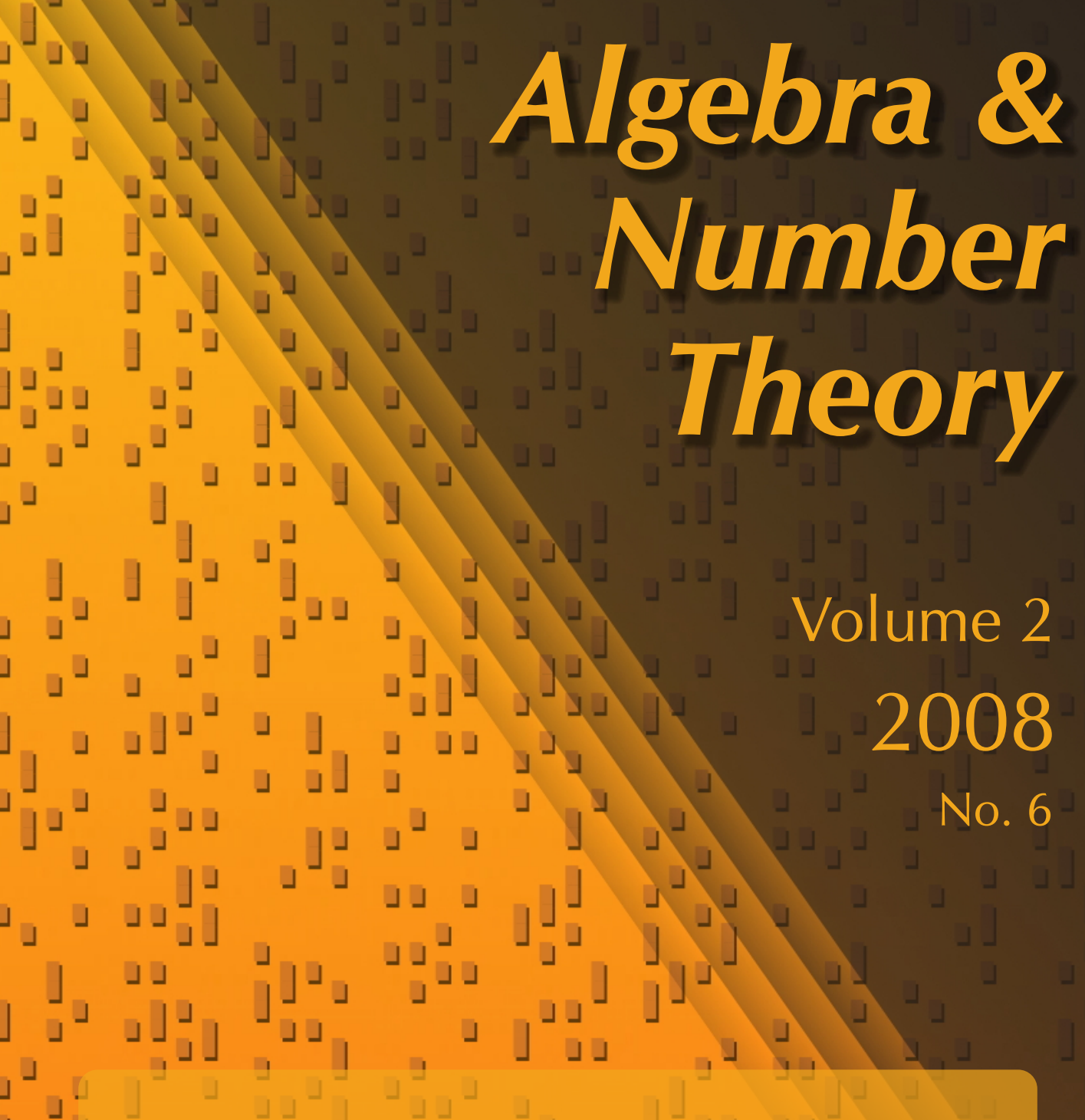

Arguments des unités de Stark et périodes de séries d'Eisenstein

\lrcorner Pierre Charollois et Henri Darmon

\lrcorner

J 


\title{
Arguments des unités de Stark et périodes de séries d'Eisenstein
}

\author{
Pierre Charollois et Henri Darmon
}

Cet article décrit une construction conjecturale (dans l'esprit du 12ème problème de Hilbert) d'unités dans des extensions abéliennes de certains corps de base qui ne sont ni totalement réels ni de type $\mathrm{CM}$. Ces corps de base possèdent un unique plongement complexe, et sont des extensions quadratiques d'un corps totalement réel $F$. On les appelle corps ATR («almost totally real»). Notre construction fait intervenir certains cycles topologiques homologiquement triviaux sur la variété modulaire de Hilbert associée à $F$. Les unités spéciales sont les images de ces cycles par une application qui repose sur l'intégration des séries d'Eisenstein de poids deux sur $\mathrm{GL}_{2}(F)$, et peut être vue comme un analogue formel des applications d'Abel-Jacobi de la théorie des cycles algébriques. On démontre que notre conjecture est compatible avec la conjecture de Stark pour les extensions ATR. Elle donne même un raffinement de la conjecture de Stark dans ce contexte, puisqu'elle fournit une formule analytique pour les arguments des unités de Stark, et pas seulement pour leurs valeurs absolues. La dernière section présente des résultats d'expériences numériques qui appuient notre conjecture.

We describe a conjectural construction (in the spirit of Hilbert's Twelfth problem) of units in abelian extensions of certain base fields which are neither totally real nor CM. These base fields are quadratic extensions with exactly one complex place of a totally real number field $F$, and are referred to as almost totally real (ATR) extensions. Our construction involves certain null-homologous topological cycles on the Hilbert modular variety attached to $F$. The special units are the images of these cycles under a map defined by integration of weight two Eisenstein series on $\mathrm{GL}_{2}(F)$. This map is formally analogous to the higher Abel-Jacobi maps that arise in the theory of algebraic cycles. We show that our conjecture is compatible with Stark's conjecture for ATR extensions; it is, however, a genuine strengthening of Stark's conjecture in this context since it gives an analytic formula for the arguments of the Stark units and not just their absolute values. The last section provides numerical evidence for our conjectures.

MSC2000: primary 11F67; secondary 11F75.

Mots-clefs: séries d'Eisenstein, périodes des formes modulaires de Hilbert, arguments des unités de

Stark, conjectures de Stark, douzième problème de Hilbert, application d'Abel-Jacobi, cohomologie du groupe modulaire de Hilbert, fonction d'Asai, valeurs spéciales de fonctions zéta partielles, Eisenstein series, periods of Hilbert modular forms, arguments of Stark units, Stark conjectures, Hilbert twelfth problem, Abel-Jacobi map, cohomology of the Hilbert modular group, Asai function, special values of partial zeta functions. 
Introduction $\quad 656$

1. Notions préliminaires 661

2. Extensions quadratiques totalement réelles et fonctions $L \quad 668$

3. Extensions quadratiques ATR et dérivées de fonctions $L \quad 670$

4. Application d'Abel-Jacobi et unités de Stark 672

5. Algorithmes 673

6. Exemples numériques 678

7. Périodes de séries d'Eisenstein. 683

$\begin{array}{ll}\text { Remerciements } & 687\end{array}$

$\begin{array}{ll}\text { Références } & 687\end{array}$

\section{Introduction}

Soit $K$ un corps de nombres, et soit

$$
S_{\infty}=\left\{v_{1}, \ldots, v_{t}\right\}
$$

l'ensemble de ses places archimédiennes. Pour simplifier les énoncés qui suivent, on supposera (dans l'introduction seulement) que le corps $K$ a pour nombre de classes 1 au sens restreint. On choisit, pour chaque place de $S_{\infty}$, un plongement réel ou complexe associé, que l'on désignera par le même symbole, et l'on pose (pour $x$ appartenant à $K^{\times}$, et $v \in S_{\infty}$ )

$$
s_{v}(x):= \begin{cases}\operatorname{signe}(v(x)) \in\{-1,1\} & \text { si } v \text { est réelle; } \\ 1 & \text { si } v \text { est complexe. }\end{cases}
$$

Soit $I$ un idéal de l'anneau des entiers $O_{K}$ de $K$, et soit $O_{K,+}^{\times}(I)$ le sous-groupe du groupe $\mathscr{O}_{K,+}^{\times}$des unités totalement positives de $\mathscr{O}_{K}$ formé des éléments qui sont congrus à 1 modulo $I$. Pour tout $a \in\left(O_{K} / I\right)$, on associe au choix de signes $s_{v_{2}}, \ldots, s_{v_{n}}$ la fonction $L$ partielle de Hurwitz :

$$
L(a, I, s):=(\mathrm{N} I)^{s} \sum_{\substack{x \in \mathbb{O}_{K} / \mathbb{O}_{K,+}^{\times}(I) \\ x \equiv a(I)}}^{\prime} s_{v_{2}}(x) \cdots s_{v_{t}}(x)\left|\mathrm{N}_{K / \mathbb{Q}}(x)\right|^{-s},
$$

où le symbole $\sum^{\prime}$ indique que la somme est à prendre sur les éléments non-nuls. Ces fonctions $L$ jouissent des propriétés suivantes :

1. La fonction $L(a, I, s)$ ne dépend que de l'image de $a$ dans le quotient

$$
\left(\mathcal{O}_{K} / I\right) / \mathcal{O}_{K,+}^{\times},
$$


sur lequel opère le groupe

$$
\mathscr{G}_{I}:=\left(\mathrm{O}_{K} / I\right)^{\times} / \mathcal{O}_{K,+}^{\times} \cdot
$$

Plus généralement, pour toute unité $\epsilon \in \mathcal{O}_{K}^{\times}$, on a

$$
L(\epsilon a, I, s)=s_{v_{2}}(\epsilon) \cdots s_{v_{t}}(\epsilon) L(a, I, s) .
$$

2. La série qui définit $L(a, I, s)$ converge absolument sur le demi-plan $\operatorname{Re}(s)>1$, et admet un prolongement méromorphe à tout le plan complexe avec au plus un pôle simple en $s=1$. Si $t \geq 2$, cette fonction est même holomorphe, et s'annule en $s=0$.

Pour toute place réelle $v \in S_{\infty}$, il existe alors une unité $\epsilon_{v} \in \mathbb{O}_{K}^{\times}$telle que

$$
s_{v}\left(\epsilon_{v}\right)=-1, \quad s_{v^{\prime}}\left(\epsilon_{v}\right)=1 \text { pour tout } v^{\prime} \neq v .
$$

De plus, la théorie du corps de classes identifie le groupe $\varphi_{I}$ avec le groupe de Galois d'une extension abélienne $H$ de $K$, appelée le corps de classes de rayon au sens restreint associé à $I$. Soit

$$
\operatorname{rec}: \mathscr{G}_{I} \rightarrow \operatorname{Gal}(H / K)
$$

l'isomorphisme de réciprocité de la théorie du corps de classes, et pour tout $v \in S_{\infty}$, soit $c_{v}$ la conjugaison complexe («élément de Frobenius») associée à la place $v$, de sorte que

$$
c_{v}= \begin{cases}\operatorname{rec}\left(\epsilon_{v}\right) & \text { si } v \text { est réelle, } \\ 1 & \text { si } v \text { est complexe. }\end{cases}
$$

On note $e$ l'ordre du groupe des racines de l'unité dans $H$ et l'on choisit une place $\tilde{v}_{1}$ de $H$ au-dessus de la place $v_{1}$.

La conjecture de Stark [1976] (voir aussi [Tate 1984]) concerne les dérivées premières de $L(a, I, s)$ en $s=0$, et peut s'énoncer comme suit :

Conjecture 1 (Stark). Pour tout $a \in\left(\mathcal{O}_{K} / I\right)$, il existe une unité $u_{a} \in \mathcal{O}_{H}^{\times}$, appelée unité de Stark associée au couple $(a, I)$, telle que

1. $L^{\prime}(a, I, 0)=\frac{1}{e} \log \left|\tilde{v}_{1}\left(u_{a}\right)\right|^{2}$;

2. $c_{v_{1}}\left(u_{a}\right)=u_{a}$;

3. Si $t \geq 3$, alors $c_{v_{2}} u_{a}=\cdots=c_{v_{t}} u_{a}=u_{a}^{-1}$;

4. Pour tout $b \in \mathscr{G}_{I}$, on $a u_{a b}=\operatorname{rec}(b)^{-1} u_{a}$.

On notera que les unités conjecturales $u_{a}$ dépendent du choix de la place $\tilde{v}_{1}$ audessus de $v_{1}$, mais seulement à conjugaison près par $\operatorname{Gal}(H / K)$.

Si $S_{\infty}-\left\{v_{1}\right\}$ possède une place complexe, la conjecture 1 est trivialement vérifiée. En effet, quand $t=2$, la quantité $L^{\prime}(a, I, 0)$ ne dépend que de $I$ et non de $a$, et s'écrit comme un multiple rationnel du logarithme d'une unité fondamentale 
de $K$. Quand $t>2$, on a de plus $L^{\prime}(a, I, 0)=0$, de sorte que la conjecture 1 est vérifiée avec $u_{a}=1$.

Par conséquent, la conjecture 1 n'a de l'intérêt que lorsque les places $v_{2}, \ldots, v_{t}$ sont toutes réelles, ce qui nous amène à distinguer deux cas.

1. Le cas totalement réel. Si la place $v_{1}$ est également réelle, le corps $K$ est totalement réel. A cause de la propriété 2 dans la conjecture 1, l'expression $\tilde{v}_{1}\left(u_{a}\right)$ appartient alors à $\mathbb{R}$. La conjecture 1 permet donc d'évaluer ce nombre, du moins au signe près. On obtient ainsi, par évaluation des dérivées en $s=0$ des séries $L(a, I, s)$, la construction analytique d'unités explicites de $H$. Les conjectures de Stark, une fois démontrées, fourniraient ainsi un élément de «théorie explicite du corps de classes» pour les corps de nombres totalement réels.

2. Le cas ATR. Si $v_{1}$ est une place complexe, on dit que $K$ est un corps de nombres ATR («almost totally real») suivant la terminologie de [Darmon et Logan 2003]. Puisque $c_{v_{1}}=1$, l'expression $\tilde{v}_{1}\left(u_{a}\right)$ n'a plus de raison d'être réelle a priori, et la conjecture 1 ne permet d'en évaluer que la valeur absolue. L'ambiguïté de signe du cas totalement réel s'avère donc plus sérieuse dans le contexte ATR, puisqu'elle porte sur l'argument de $\tilde{v}_{1}\left(u_{a}\right)$, un élément de $\mathbb{R} /(2 \pi \mathbb{Z})$. On est amené à poser la question suivante qui peut servir de motivation pour cet article.

Question 1. Existe-t-il une formule analytique explicite pour l'expression $\tilde{v}_{1}\left(u_{a}\right)$ qui apparaît dans la conjecture 1, lorsque $K$ est ATR?

Une réponse affirmative à cette question fournirait une solution du douzième problème de Hilbert pour les extensions ATR.

Dans le premier cas intéressant où $K$ est un corps cubique complexe, cette question a été considérée dans [Dasgupta 1999] et dans [Dummit et al. 2004] où la conjecture de Stark est étudiée numériquement, et un progrès décisif a été accompli dans [Ren et Sczech 2008].

Le présent article se penche sur la Question 1 lorsque le corps $K$ est une extension quadratique d'un corps totalement réel $F$, et lorsque le corps de rayon $H$ est remplacé par un certain sous-corps - le corps de classes d'anneau ( $\ll$ ring class field») associé à $I$ et $K / F$ - dont la définition sera rappelée dans la section 1 .

Pour motiver notre approche, examinons ce qui se passe dans le cas le plus simple où $F=\mathbb{Q}$, où $K$ est un corps quadratique imaginaire de nombre de classes 1 , et où $I=(m)$ est un idéal rationnel engendré par $m \in \mathbb{Z}$. Au lieu de porter sur les séries partielles de Hurwitz, les conjectures de cet article vont plutôt porter sur les sommes

$$
\sum_{r \in(\mathbb{Z} / m \mathbb{Z})} L(a r, I, s)=: L(M, s)=(\mathrm{N} M)^{s} \sum_{x \in M / \mathbb{O}_{K,+}^{\times}(I)}^{\prime}\left|\mathrm{N}_{K / \mathbb{Q}}(x)\right|^{-s},
$$


où

$$
M=\left\{x \in \mathbb{O}_{K}, \text { tel qu'il existe } r \in \mathbb{Z} \text { avec } x \equiv \text { ar } \quad(\bmod I)\right\} .
$$

Le module $M$ est un réseau dans $K \subset \mathbb{C}$, et, quand $a$ appartient à $\mathscr{G}_{I}$, il forme même un module projectif sur l'ordre $O_{I}:=\mathbb{Z}+I O_{K}$. La série $L(M, s)$ ne dépend que de la classe d'homothétie de ce réseau; elle est donc déterminée par l'invariant $\tau=$ $\omega_{2} / \omega_{1}$, où $\left(\omega_{1}, \omega_{2}\right)$ est une $\mathbb{Z}$-base de $M$ choisie de telle manière que $\tau$ appartienne au demi-plan de Poincaré $\mathscr{H}$. La formule limite de Kronecker fournit les premiers termes du développement de $L(M, s)$ en $s=0$ en la reliant au logarithme de la fonction $\eta$ de Dedekind :

$$
L(M, s)=-\frac{1}{2}-\frac{1}{2}\left(c_{I}+\log \operatorname{Im}(\tau)+4 \log |\eta(\tau)|\right) s+O\left(s^{2}\right),
$$

où $c_{I}$ est une constante qui ne dépend que de $I$ et pas de $M$. À des facteurs parasites près, les dérivées premières $L^{\prime}(M, 0)$ sont donc fournies par l'expression $\log |\eta(\tau)|$. Or, comme $\tau$ appartient à $\mathscr{H} \cap K$, les produits d'expressions de la forme $\eta(\tau)$ donnent lieu aux unités elliptiques, que l'on sait être des unités dans des extensions abéliennes du corps $K$ grâce à la théorie de la multiplication complexe. Plus précisément, pour $\tau_{1}, \tau_{2} \in \mathscr{H} \cap K$, les expressions de la forme

$$
u\left(\tau_{1}, \tau_{2}\right):=\eta\left(\tau_{2}\right) / \eta\left(\tau_{1}\right)
$$

sont des nombres algébriques, et leurs puissances 24-èmes sont des unités dans des extensions abéliennes de $K$. C'est ainsi que les propriétés de la fonction $\eta$ et la théorie de la multiplication complexe permettent non seulement de démontrer la conjecture de Stark dans le cas où $K$ est quadratique imaginaire, mais apportent aussi une réponse à la Question 1 dans ce cas.

Dans la généralisation «traditionnelle» de la théorie de la multiplication complexe proposée par Hilbert et son école, puis développée rigoureusement par Shimura et Taniyama, on est amené à remplacer $\mathbb{Q}$ par un corps $F$ totalement réel de degré $n>1$ (de sorte que $F \otimes_{\mathbb{Q}} \mathbb{R}=\mathbb{R}^{n}$ ), et les formes modulaires classiques par des formes modulaires de Hilbert. Celles-ci correspondent à des fonctions holomorphes sur $\mathscr{H}^{n}$, invariantes (à un facteur d'automorphie près) sous l'action naturelle de $\mathbf{S L}_{2}\left(O_{F}\right)$. Les corps quadratiques imaginaires sont remplacés par des extensions quadratiques $K$ de $F$ de type CM, munies d'une identification $\Phi: K \otimes_{F} \mathbb{R}^{n} \rightarrow \mathbb{C}^{n}$. La théorie de la multiplication complexe affirme alors que les valeurs de certaines fonctions modulaires de Hilbert (quotients de formes modulaires de même poids, possédant des développements de Fourier rationnels) en des points $\tau \in \Phi(K) \cap \mathscr{H}^{n}$ engendrent des extensions abéliennes du corps reflex $\tilde{K}$ associé à $(K, \Phi)$. Cette théorie possède deux inconvénients du point de vue de la Question 1 :

(a) elle ne permet d'aborder la «théorie du corps de classes explicite» que pour les corps de base de type CM; 
(b) elle ne permet pas d'obtenir facilement des unités dans des extensions abéliennes de $\tilde{K}$, les unités modulaires n'ayant pas de généralisation évidente pour les formes modulaires de Hilbert. En effet, quand $n>1$, le faisceau structural sur l'espace complexe analytique $X:=\mathbf{S L}_{2}\left(\mathscr{O}_{F}\right) \backslash \mathscr{H}^{n}$ ne possède pas de sections globales non-nulles. La relation entre la théorie de ShimuraTaniyama et les conjectures de Stark pour les corps CM (à supposer qu'il y en ait une) reste donc à élucider. (Voir par exemple [de Shalit et Goren 1997] et [Goren et Lauter 2007].)

Pour aborder la Question 1 lorsque $K$ est une extension quadratique ATR d'un corps $F$ totalement réel de degré $n>1$, il faut relever le nombre réel $\log \left|\tilde{v}_{1}\left(u_{a}\right)\right|$ en un nombre complexe $\log \tilde{v}_{1}\left(u_{a}\right) \in \mathbb{C} / 2 i \pi \mathbb{Z}$. Au vu de la formule limite de Kronecker (4) et de la discussion précédente, il s'agit donc de généraliser l'expression

$$
\log \eta(\tau)=\log |\eta(\tau)|+i \arg \eta(\tau) \in \mathbb{C} / 2 i \pi \mathbb{Z}
$$

à un cadre où les formes modulaires classiques sont remplacées par des formes modulaires de Hilbert sur $F$. C'est ce qui a été entrepris dans la thèse du premier auteur, qui part de l'identité classique $\mathrm{d} \log \eta(z)=-i \pi E_{2}(z) d z$, où $E_{2}$ est la série d'Eisenstein définie par

$$
E_{2}(z)=-\frac{1}{12}+2 \sum_{n \geq 1} \sigma_{1}(n) e^{2 i \pi n z}, \quad \text { avec } \sigma_{1}(n)=\sum_{d \mid n} d .
$$

La formule (5) peut donc se réécrire en prenant le logarithme complexe des deux côtés :

$$
\log \left(u\left(\tau_{1}, \tau_{2}\right)\right)=-i \pi \int_{\tau_{1}}^{\tau_{2}} E_{2}(z) d z \in \mathbb{C} / 2 i \pi \mathbb{Z} .
$$

Or, si les unités modulaires n'admettent pas d'analogue évident en dimension supérieure, les séries d'Eisenstein, elles, se généralisent sans difficulté à ce contexte. La section 1.1 rappelle la définition de la série d'Eisenstein $E_{2}$ de poids $(2, \ldots, 2)$ sur $\mathbf{S L}_{2}\left(O_{F}\right)$. Celle-ci donne lieu à une $n$-forme différentielle $\omega_{E_{2}}$ holomorphe sur l'espace analytique $X$.

La démarche suggérée par Charollois [2004] consiste essentiellement à remplacer les valeurs de $\log \eta(\tau)$ par des intégrales de $\omega_{E_{2}}$ sur des cycles appropriés de dimension réelle $n$ sur $X$. Plus précisément, le présent article associe à tout $\tau \in \mathscr{H} \cap v_{1}(K)$ un cycle fermé $\Delta_{\tau}$ de dimension réelle $(n-1)$ sur $X$. En faisant abstraction pour le moment des phénomènes liés à la présence possible de torsion dans la cohomologie de $X$, on démontre que ces cycles sont homologues à zéro. Autrement dit, il existe une chaîne différentiable $C_{\tau}$ de dimension $n$ sur $X$ telle que

$$
\partial C_{\tau}=\Delta_{\tau}
$$


Cela permet de définir un invariant canonique $J_{\tau} \in \mathbb{C} / 2 i \pi \mathbb{Z}$ en intégrant un multiple approprié de $\omega_{E_{2}}$ sur $C_{\tau}$. La contribution la plus importante de cet article est la conjecture 4.1, qui relie les invariants $J_{\tau}$ à l'expression $\log \tilde{v}_{1}\left(u_{a}\right)$ dont la partie réelle apparaît dans la conjecture 1. La conjecture 4.1 apporte ainsi un élément de réponse à la Question 1.

La définition des invariants $J_{\tau}$ s'appuie de façon essentielle sur la thèse du premier auteur [Charollois 2004]. Elle est aussi à rapprocher de deux autres travaux antérieurs :

1. L'article [Darmon et Logan 2003], où les séries d'Eisenstein sur $\mathbf{G L}_{2}(F)$ du présent article sont remplacées par des formes modulaires de Hilbert cuspidales de poids $(2, \ldots, 2)$. Dans les cas les plus concrets qui ont pu être testés numériquement, ces formes sont associées à des courbes elliptiques définies sur $F$. L'invariant $J_{\tau}$ défini dans ce contexte semble alors permettre la construction de points sur ces courbes définis sur certaines extensions abéliennes de $K$.

2. L'article [Darmon et Dasgupta 2006] peut être considéré comme une variante $p$-adique, avec $F=\mathbb{Q}$, des constructions principales du présent article, le rôle de notre $v_{1}$ y étant joué par une place non-archimédienne $p$. L'extension $K$ est alors un corps quadratique réel dans lequel le nombre premier $p$ est inerte. Les séries d'Eisenstein de poids 2 sur certains sous-groupes de congruence de $\mathbf{S L}_{2}(\mathbb{Z})$, réinterprétées convenablement comme des «formes modulaires de Hilbert» sur $\mathscr{H}_{p} \times \mathscr{H}$, où $\mathscr{H}_{p}=\mathbf{P}_{1}\left(\mathbb{C}_{p}\right)-\mathbf{P}_{1}\left(\mathbb{Q}_{p}\right)$ est le demi-plan $p$-adique, donnent alors lieu à des invariants $p$-adiques $J_{\tau} \in \mathbb{C}_{p}$ associés à un élément $\tau \in \mathscr{H}_{p} \cap K$. Ces invariants correspondent conjecturalement à des $p$-unités dans des extensions abéliennes de $K$.

En se plaçant dans un cadre classique où l'on dispose de notions topologiques et analytiques générales (homologie et cohomologie singulière, théorie de Hodge), le présent article mène à une clarification conceptuelle des différentes constructions de «points et unités de Stark-Heegner» proposées jusqu'à présent dans la littérature. (Voir [Darmon et Logan 2003] et [Darmon et Dasgupta 2006], ainsi que le cadre traité originalement dans [Darmon 2004] ou les généralisations formulées dans [Trifković 2006] et [Greenberg 2008].) Le présent article peut très bien servir d'introduction aux travaux sur les points de Stark-Heegner cités en référence, bien qu'il ait été rédigé après ceux-ci.

\section{Notions préliminaires}

1.1. Séries d'Eisenstein. Soit $F$ un corps totalement réel de degré $n>1$ et $S_{F}:=$ $\left\{v_{1}, \ldots, v_{n}\right\}$ son ensemble de places archimédiennes. On désigne par $\mathcal{O}_{F}$ l'anneau des entiers de $F$, par $d_{F}$ son discriminant, et par $R_{F}$ le régulateur de $F$. 
On supposera dans la suite de cet article que $F$ a nombre de classes 1 au sens restreint, de sorte qu'il existe pour tout $1 \leq j \leq n$ une unité $\epsilon^{(j)} \in \mathbb{O}_{F}^{\times}$avec

$$
v_{j}\left(\epsilon^{(j)}\right)<0, \quad v_{k}\left(\epsilon^{(j)}\right)>0 \quad \text { si } k \neq j .
$$

Pour tout $a \in F$, on note $a_{j}:=v_{j}(a)$ son image dans $\mathbb{R}$ par le plongement $v_{j}$, et $A_{j}=\left(\begin{array}{ll}a_{j} & b_{j} \\ c_{j} & d_{j}\end{array}\right)$ l'image dans $\mathbf{S L}_{2}(\mathbb{R})$ d'une matrice $A=\left(\begin{array}{ll}a & b \\ c & d\end{array}\right)$ de $\mathbf{S L}_{2}(F)$. On identifiera librement $a$ avec le $n$-uplet $\left(a_{1}, \ldots, a_{n}\right)$ et $A$ avec $\left(A_{1}, \ldots, A_{n}\right)$. On obtient ainsi une action par homographies du groupe modulaire de Hilbert $\Gamma:=$ $\mathbf{S L}_{2}\left(\mathscr{O}_{F}\right)$ sur le produit $\mathscr{H}_{1} \times \cdots \times \mathscr{H}_{n}$ de $n$ copies du demi-plan de Poincaré. Le quotient analytique

$$
X:=\Gamma \backslash\left(\mathscr{H}_{1} \times \cdots \times \mathscr{H}_{n}\right)
$$

s'identifie avec les points complexes d'un ouvert de Zariski d'une variété algébrique projective lisse : la variété modulaire de Hilbert $X_{F}$ associée au corps $F$.

Une forme modulaire de Hilbert de poids $(2, \ldots, 2)$ pour $\Gamma$ est une fonction holomorphe $f\left(z_{1}, \ldots, z_{n}\right)$ sur $\mathscr{H}_{1} \times \cdots \times \mathscr{H}_{n}$ telle que la forme différentielle

$$
\omega_{f}:=f\left(z_{1}, \ldots z_{n}\right) d z_{1} \wedge \cdots \wedge d z_{n}
$$

soit invariante sous l'action de $\Gamma$. Autrement dit, pour tout $\left(\begin{array}{ll}a & b \\ c & d\end{array}\right) \in \Gamma$, on exige que

$$
f\left(\frac{a_{1} z_{1}+b_{1}}{c_{1} z_{1}+d_{1}}, \ldots, \frac{a_{n} z_{n}+b_{n}}{c_{n} z_{n}+d_{n}}\right)=\left(c_{1} z_{1}+d_{1}\right)^{2} \cdots\left(c_{n} z_{n}+d_{n}\right)^{2} f\left(z_{1}, \ldots, z_{n}\right) .
$$

Lorsque $n>1$, une telle fonction possède, d'après le principe de Koecher, un développement en série de Fourier à l'infini de la forme

$$
f\left(z_{1}, \ldots, z_{n}\right)=a_{f}(0)+\sum_{\mu \in \mathbb{O}_{F}, \mu \gg 0} a_{f}(\mu) e^{2 i \pi\left(\frac{\mu_{1}}{\delta_{1}} z_{1}+\cdots+\frac{\mu_{n}}{\delta_{n}} z_{n}\right)},
$$

où $\delta$ désigne un générateur totalement positif de la différente de $F / \mathbb{Q}$.

La série d'Eisenstein holomorphe $E_{2}$ de poids 2 se définit sur $\mathscr{H}_{1} \times \cdots \times \mathscr{H}_{n}$ par le développement en série de Fourier suivant :

$$
E_{2}\left(z_{1}, \ldots, z_{n}\right)=\zeta_{F}(-1)+2^{n} \sum_{\mu \in \mathscr{O}_{F}, \mu \gg 0} \sigma_{1}(\mu) e^{2 i \pi\left(\frac{\mu_{1}}{\delta_{1}} z_{1}+\cdots+\frac{\mu_{n}}{\delta_{n}} z_{n}\right)},
$$

où, pour un entier $k$ donné, on a posé

$$
\sigma_{k}(\mu)=\sum_{(\nu) \mid(\mu)}\left|N_{F / \mathbb{Q}}(v)\right|^{k},
$$


la sommation portant sur les idéaux (principaux) entiers $(v)$ qui divisent $(\mu)$. La fonction $E_{2}\left(z_{1}, \ldots, z_{n}\right)$ est une forme modulaire de Hilbert de poids $(2, \ldots, 2)$ pour $\Gamma$ (voir [van der Geer 1988, chap. I.6]).

On se donne des coordonnées réelles $x_{j}, y_{j}$ sur $X$ en posant $z_{j}=x_{j}+i y_{j}$, et l'on définit à partir de $E_{2}$ une forme différentielle invariante $\omega_{\text {Eis }}$ en posant

$$
\omega_{\text {Eis }}:= \begin{cases}\frac{(2 i \pi)^{2}}{\sqrt{d_{F}}} \omega_{E_{2}}+\frac{R_{F}}{2}\left(\frac{d z_{1} \wedge d \bar{z}_{1}}{y_{1}^{2}}-\frac{d z_{2} \wedge d \bar{z}_{2}}{y_{2}^{2}}\right) & \text { si } n=2, \\ \frac{(2 i \pi)^{n}}{\sqrt{d_{F}}} \omega_{E_{2}} & \text { si } n \geq 3 .\end{cases}
$$

La $n$-forme différentielle $\omega_{\text {Eis }}$ est fermée et, quand $n \geq 3$, elle est holomorphe. On va s'intéresser à sa classe dans la cohomologie $H^{n}(X, \mathbb{C})$ formée à partir du complexe de De Rham des formes différentielles $C^{\infty}$ sur $X$. Ce groupe de cohomologie est muni d'une action des opérateurs de Hecke $T_{\lambda}$, où les $\lambda$ parcourent les idéaux de $O_{F}$. La forme différentielle $\omega_{\text {Eis }}$ est vecteur propre pour ces opérateurs. Plus précisément, on a

$$
T_{\lambda}\left(\omega_{\text {Eis }}\right)=\sigma_{1}(\lambda) \omega_{\text {Eis }} .
$$

Pour tout $1 \leq j \leq n$, on dispose également d'une involution $T_{v_{j}}$ sur l'espace réelanalytique $X$ associée à la place $v_{j}$ («opérateur de Hecke à l'infini»). Celle-ci se définit en posant

$$
T_{v_{j}}\left(z_{1}, \ldots, z_{n}\right):=\left(\epsilon_{1}^{(j)} z_{1}, \ldots, \epsilon_{j}^{(j)} \bar{z}_{j}, \ldots, \epsilon_{n}^{(j)} z_{n}\right) .
$$

On appelle $T_{v_{j}}^{*}$ l'involution sur $H^{n}(X, \mathbb{C})$ qui s'en déduit par pullback sur les formes différentielles. Les $n$ opérateurs $T_{v_{j}}^{*}$ et les opérateurs de Hecke $T_{\lambda}$ engendrent une algèbre commutative $\mathbf{T}$ sur $\mathbb{Z}$.

On aura besoin dans la suite de certaines fonctions qui joueront le rôle de primitives de $E_{2}$. On introduit pour cela la fonction $h$ de Asai [1970] définie sur $\mathscr{H}^{n}$ par

$$
\begin{aligned}
h(z)=\frac{4(-\pi)^{n} \zeta_{F}(-1)}{R_{F} \sqrt{d_{F}}} & y_{1} \cdots y_{n} \\
& \quad+\frac{4 \sqrt{d_{F}}}{2^{n} R_{F}} \sum_{\mu \in O_{F}}^{\prime} \sigma_{-1}(\mu) e^{2 i \pi\left(\frac{\mu_{1}}{\delta_{1}} x_{1}+\left|\frac{\mu_{1}}{\delta_{1}}\right| i y_{1}+\cdots+\frac{\mu_{n}}{\delta_{n}} x_{n}+\left|\frac{\mu_{n}}{\delta_{n}}\right| i y_{n}\right) .}
\end{aligned}
$$

Il sera plus commode de travailler avec

$$
\tilde{h}(z):=\lambda_{F} h(z), \quad \text { où } \lambda_{F}:=4^{n-1} R_{F},
$$

de sorte que

$$
\begin{aligned}
\tilde{h}(z)=\frac{(-4 \pi)^{n} \zeta_{F}(-1)}{\sqrt{d_{F}}} & y_{1} \cdots y_{n} \\
& +2^{n} \sqrt{d_{F}} \sum_{\mu \in O_{F}}^{\prime} \sigma_{-1}(\mu) e^{2 i \pi\left(\frac{\mu_{1}}{\delta_{1}} x_{1}+\left|\frac{\mu_{1}}{\delta_{1}}\right| i y_{1}+\cdots+\frac{\mu_{n}}{\delta_{n}} x_{n}+\left|\frac{\mu_{n}}{\delta_{n}}\right| i y_{n}\right) .}
\end{aligned}
$$


Les fonctions $h(z)$ et $\tilde{h}(z)$ jouissent des propriétés suivantes :

1. Elles sont harmoniques par rapport à chacune des variables $z_{j}, 1 \leq j \leq n$.

2. Elles satisfont les formules de transformation

$$
\begin{aligned}
& h(A z)=h(z)-\log \left(\left|c_{1} z_{1}+d_{1}\right|^{2} \cdots\left|c_{n} z_{n}+d_{n}\right|^{2}\right), \\
& \tilde{h}(A z)=\tilde{h}(z)-\lambda_{F} \log \left(\left|c_{1} z_{1}+d_{1}\right|^{2} \cdots\left|c_{n} z_{n}+d_{n}\right|^{2}\right) .
\end{aligned}
$$

3. On a

$$
\begin{gathered}
\frac{\partial^{n} \tilde{h}\left(z_{1}, \ldots, z_{n}\right)}{\partial z_{1} \cdots \partial z_{n}} d z_{1} \wedge \cdots \wedge d z_{n}=\frac{(2 i \pi)^{n}}{\sqrt{d_{F}}} \omega_{E_{2}} \\
\frac{\partial^{n} \tilde{h}\left(z_{1}, \ldots, z_{n}\right)}{\partial z_{1} \cdots \partial \bar{z}_{j} \cdots \partial z_{n}} d z_{1} \wedge \cdots d \bar{z}_{j} \cdots \wedge d z_{n}=T_{v_{j}}^{*}\left(\frac{(2 i \pi)^{n}}{\sqrt{d_{F}}} \omega_{E_{2}}\right) .
\end{gathered}
$$

Toutes ces formules se vérifient par un calcul direct, sauf (11) et (12). Pour ces dernières, voir [Asai 1970], théorème 4.

Lemme 1.1. La forme $\left(\mathrm{Id}+T_{v_{j}}^{*}\right) \omega_{\text {Eis }}$ est exacte.

Démonstration. Supposons que $j=1$ pour fixer les idées et alléger les notations. À partir de la fonction $\tilde{h}$, on définit la $(n-1)$ forme différentielle sur $\mathscr{H}^{n}$ :

$$
\eta=\frac{\partial^{n-1} \tilde{h}\left(z_{1}, \ldots, z_{n}\right)}{\partial z_{2} \cdots \partial z_{n}} d z_{2} \wedge \cdots \wedge d z_{n}
$$

Quand $n>2$, la formule (12) montre que $\eta$ est invariante sous $\Gamma$, et correspond donc à une $(n-1)$-forme différentielle sur $X$. Parce que $\tilde{h}$ est harmonique, cette forme est de plus holomorphe par rapport aux variables $z_{2}, \ldots, z_{n}$, d'où la formule

$$
d \eta=\left(\frac{\partial^{n} \tilde{h}}{\partial z_{1} \cdots \partial z_{n}} d z_{1} \wedge \cdots \wedge d z_{n}+\frac{\partial^{n} \tilde{h}}{\partial \overline{z_{1}} \partial z_{2} \cdots \partial z_{n}} d \overline{z_{1}} \wedge d z_{2} \wedge \cdots \wedge d z_{n}\right) .
$$

Le lemme résulte alors de (13) et de (14) avec $j=1$.

Dans le cas où $n=2$, la forme $\eta$ définie par (15) n'est plus $\Gamma$-invariante. En effet, si $A=\left(\begin{array}{ll}a & b \\ c & d\end{array}\right) \in \Gamma$, on a

$$
A^{*}(\eta)=\eta-4 R_{F} \frac{c_{2}}{c_{2} z_{2}+d_{2}} d z_{2}
$$

Il convient alors de modifier la définition de $\eta$ en (15) en posant cette fois

$$
\eta^{\prime}:=\left(\frac{\partial \tilde{h}\left(z_{1}, z_{2}\right)}{\partial z_{2}}-\frac{2 R_{F}}{i y_{2}}\right) d z_{2}
$$


On déduit de l'identité (12) que $\eta^{\prime}$ est invariante sous $\Gamma$. La formule (16) s'adapte sans difficulté à condition d'ajouter la contribution de

$$
d\left(\frac{-2 R_{F}}{i y_{2}} d z_{2}\right)=-R_{F} \frac{d z_{2} \wedge d \bar{z}_{2}}{y_{2}^{2}} .
$$

On obtient

$$
\left(\mathrm{Id}+T_{v_{1}}^{*}\right) \omega_{\text {Eis }}=d \eta^{\prime},
$$

puisque la forme $d z_{1} \wedge d \bar{z}_{1} / y_{1}^{2}$ est quant à elle dans le noyau de $\mathrm{Id}+T_{v_{1}}^{*}$. C'est ce calcul qui justifie le terme supplémentaire apparaissant dans la définition (8) de $\omega_{\text {Eis }}$ lorsque $n=2$.

Pour $m \geq 0$, on appelle $C_{m}^{0}(X)$ le groupe engendré par les combinaisons linéaires formelles à coefficients dans $\mathbb{Z}$ des cycles différentiables fermés de dimension réelle $m$ sur $X$. On définit le groupe des périodes de $\omega_{\text {Eis }}$ par

$$
\Lambda_{\text {Eis }}:=\left\{\int_{C} \omega_{\text {Eis }} \text { pour } C \in C_{n}^{0}(X)\right\} \subset \mathbb{C} .
$$

Proposition 1.2. Le groupe $\Lambda_{\text {Eis }}$ est un sous-groupe de $\mathbb{C}$ de rang un, commensurable avec $(2 i \pi)^{n} \mathbb{Z}$.

Démonstration. Cette proposition se démontre en trois parties.

(a) Le groupe $\Lambda_{\text {Eis }}$ est un sous-ensemble discret de $\mathbb{C}$. La théorie de Harder [1975] (voir aussi le théorème 6.3, chap. III, $\$ 7$ de [Freitag 1990] avec $m=n$ ) fournit la décomposition

$$
H^{n}(X, \mathbb{C})=H_{\text {univ }}^{n}(X, \mathbb{C}) \oplus H_{\text {Eis }}^{n}(X, \mathbb{C}) \oplus H_{\text {cusp }}^{n}(X, \mathbb{C}),
$$

où $H_{\text {univ }}^{n}(X, \mathbb{C})$ provient des formes différentielles $\mathbf{S L}_{2}(\mathbb{R})^{n}$-invariantes, $H_{\text {Eis }}^{n}(X, \mathbb{C})$ est l'espace vectoriel de dimension 1 engendré par $\left[\omega_{\text {Eis }}\right]$, et $H_{\text {cusp }}^{n}(X, \mathbb{C})$ provient des formes modulaires cuspidales de poids $(2, \ldots, 2)$ sur $X$. La décomposition (18) est respectée par l'algèbre de Hecke T, et les éléments $\omega \in H_{\text {Eis }}^{n}$ sont caractérisés par les propriétés

$$
T_{v_{j}}^{*} \omega=-\omega, \quad T_{\lambda} \omega=(\mathrm{N} \lambda+1) \omega, \quad \text { pour tout } \lambda \triangleleft \mathcal{O}_{F} .
$$

Il en résulte que la projection naturelle $H^{n}(X, \mathbb{C}) \rightarrow H_{\text {Eis }}^{n}(X, \mathbb{C})$ issue de (18) est décrite par un idempotent $\pi_{\text {Eis }} \in \mathbf{T} \otimes \mathbb{Q}$. Soit $\Lambda$ l'image naturelle de $H_{n}(X, \mathbb{Z})$ dans le dual $H^{n}(X, \mathbb{C})^{\vee}:=\operatorname{Hom}\left(H^{n}(X, \mathbb{C}), \mathbb{C}\right)$ par l'application des périodes. C'est un sous-groupe discret stable pour l'action de $\mathbf{T}$. On a de plus

$$
\Lambda_{\text {Eis }}=\left\langle\omega_{\text {Eis }}, \pi_{\text {Eis }}(\Lambda)\right\rangle,
$$

où $\langle$,$\rangle désigne l'accouplement naturel entre H^{n}(X, \mathbb{C})$ et son dual. Or on a

$$
\pi_{\text {Eis }}(\Lambda) \subset \frac{1}{t} \Lambda \cap H_{\text {Eis }}^{n}(X, \mathbb{C})^{\vee},
$$


où $t$ est un entier tel que $t \pi_{\text {Eis }}$ appartient à $\mathbf{T}$. Par conséquent $\pi_{\text {Eis }}(\Lambda)$ est un sousgroupe discret de $H_{\text {Eis }}^{n}(X, \mathbb{C})^{\vee}$, ce qui implique que $\Lambda_{\text {Eis }}$ est lui aussi discret.

(b) Le groupe $\Lambda_{\text {Eis }}$ est contenu dans $(2 i \pi)^{n} \mathbb{R}$. En effet, le lemme 1.1 implique que $T_{v_{1}}^{*} \cdots T_{v_{n}}^{*}\left(\left[\omega_{\mathrm{Eis}}\right]\right)=(-1)^{n}\left[\omega_{\mathrm{Eis}}\right]$. Par ailleurs, un calcul direct montre que $T_{v_{1}}^{*} \cdots T_{v_{n}}^{*}\left(\left[\omega_{\mathrm{Eis}}\right]\right)=\left[\bar{\omega}_{\mathrm{Eis}}\right]$. On en déduit que $\left[\bar{\omega}_{\mathrm{Eis}}\right]=(-1)^{n}\left[\omega_{\mathrm{Eis}}\right]$. Les périodes de $\omega_{\text {Eis }}$ appartiennent donc bien à $(2 i \pi)^{n} \mathbb{R}$.

(c) Les parties (a) et (b) montrent que $\Lambda_{\text {Eis }}$ est de rang au plus un. Pour montrer qu'il est non-trivial et déterminer sa classe de commensurabilité, il suffit de calculer une période non-nulle de $\omega_{\text {Eis }}$. Pour cela, on fixe $Y_{1}, \ldots, Y_{n} \in \mathbb{R}_{>0}$ et l'on considère les droites horizontales $L_{j} \subset \mathscr{H}_{j}$ formées des $z_{j}$ dont la partie imaginaire est égale à $Y_{j}$. La région $R_{\infty}:=L_{1} \times \cdots \times L_{n}$ est préservée par le sous-groupe des translations $\Gamma_{\infty} \subset \Gamma$. Soit $D_{\infty}$ un domaine fondamental compact pour cette action. Son image dans $X$ est un cycle fermé de dimension $n$. La forme différentielle $\omega_{\text {Eis }}$ peut s'intégrer terme à terme sur $D_{\infty}$ à partir de la formule (7). Seul le terme constant dans la définition de $E_{2}$ apporte une contribution non-nulle à l'intégrale, puisque les autres termes sont des multiples de caractères non-triviaux de $R_{\infty} / \Gamma_{\infty}$. Comme le volume de $R_{\infty} / \Gamma_{\infty}$ est égal à $\sqrt{d_{F}}$, on en déduit que

$$
\int_{D_{\infty}} \omega_{\text {Eis }}=(2 i \pi)^{n} \zeta_{F}(-1) \text {. }
$$

Ceci achève la démonstration, puisque $\zeta_{F}(-1)$ appartient à $\mathbb{Q}^{\times}$.

1.2. Extensions quadratiques et séries $L$ associées. Soit $K$ une extension quadratique de $F$. Pour chaque $1 \leq j \leq n$, la $\mathbb{R}$-algèbre $K \otimes_{F, v_{j}} \mathbb{R}$ est isomorphe soit à $\mathbb{C}$, soit à $\mathbb{R} \oplus \mathbb{R}$. On fixe une telle identification, que l'on appelle aussi $v_{j}$ par abus de notation. Lorsque $K$ est un corps CM, la donnée de $\left(v_{1}, \ldots, v_{n}\right)$ correspond au choix d'un type $\mathrm{CM}$ associé à $K$. On sait à quel point cette donnée supplémentaire joue un rôle important dans la théorie de la multiplication complexe pour les extensions $\mathrm{CM}$ de $F$.

On munit les $\mathbb{R}$-espaces vectoriels $\mathbb{C}$ et $\mathbb{R} \oplus \mathbb{R}$ de l'orientation standard dans laquelle une orientation positive est assignée aux bases $(1, i)$ et $((1,0),(0,1))$ de $\mathbb{C}$ et $\mathbb{R} \oplus \mathbb{R}$ respectivement. Une base de $K$ (vu comme espace vectoriel sur $F$ de dimension 2) est alors dite positive si ses images dans $\mathbb{C}$ et $\mathbb{R} \oplus \mathbb{R}$ par les plongements $v_{j}$ sont orientées positivement. On remarque en particulier que la base $(1, \tau)$ de $K$ sur $F$ est positive si et seulement si :

1. on a $\tau_{j}^{\prime}>\tau_{j}$ pour toute place réelle $v_{j}$ de $F$;

2. la partie imaginaire de $\tau_{j}$ est strictement positive pour toute place complexe $v_{j}$.

Soit $I$ un idéal de l'anneau $O_{F}$. On se permet de désigner par le même symbole l'idéal $I O_{K}$ de $K$. 
On maintiendra tout au long de cet article l'hypothèse que $F$ a pour nombre de classes 1 au sens étroit. Par contre, il est souhaitable de ne pas avoir à faire d'hypothèse semblable sur le corps $K$. On généralise la définition (2) du groupe $\mathscr{G}_{I}$ de l'introduction, en le définissant comme un quotient approprié du groupe $\mathbf{A}_{K}^{\times}$des idèles de $K$. Pour chaque place non-archimédienne $v$ de $K$, on appelle $\mathcal{O}_{v}$ l'anneau des entiers du corps local $K_{v}$, et l'on pose

avec

$$
\varphi_{I}:=\mathbf{A}_{K}^{\times} /\left(\prod_{v} U_{v}\right) K^{\times},
$$

$$
U_{v}= \begin{cases}\mathbb{R}_{>0}^{\times} & \text {si } v \text { est réelle; } \\ \mathbb{C}^{\times} & \text {si } v \text { est complexe; } \\ \mathcal{O}_{v}^{\times} & \text {si } v \nmid I ; \\ 1+I O_{v} & \text { si } v \mid I .\end{cases}
$$

Comme dans l'introduction, la loi de réciprocité du corps de classes donne un isomorphisme rec : $\mathscr{G}_{I} \rightarrow \mathrm{Gal}(H / K)$, où $H$ est le corps de classes de rayon de $K$ au sens restreint associé à $I$.

Le sous-corps $F$ permet d'introduire un sous-groupe $\mathscr{G}_{I}^{+} \subset \mathscr{G}_{I}$, défini comme l'image naturelle dans $\varphi_{I}$ du groupe $\mathbf{A}_{F}^{\times}$. Le sous-corps $H_{I}$ de $H$ fixé par rec( $\left(\varphi_{I}^{+}\right)$ s'appelle le corps de classes d'anneau associé à $I$ et $K / F$. On a donc l'isomorphisme de réciprocité

$$
\text { rec : } G_{I} \rightarrow \operatorname{Gal}\left(H_{I} / K\right), \quad \text { où } G_{I}:=\mathbf{A}_{K}^{\times} /\left(\mathbf{A}_{F}^{\times} \prod_{v} U_{v} K^{\times}\right) .
$$

Un $\mathscr{O}_{F}$-ordre de $K$ est un sous-anneau de $K$ qui contient $\mathscr{O}_{F}$ et qui est localement libre de rang 2 sur $O_{F}$ (donc libre, puisque $h(F)=1$ ). On désigne par

$$
\mathfrak{O}_{I}:=\mathscr{O}_{F}+I \mathfrak{O}_{K}
$$

l'ordre de $K$ de conducteur $I$, et l'on appelle

$$
\hat{\mathrm{O}}_{I}=\prod_{v \nmid \infty} \hat{O}_{I} \otimes \hat{O}_{F, v}
$$

son adélisation. On a alors

$$
G_{I}=\mathbf{A}_{K}^{\times} /\left(\hat{O}_{I}^{\times} \prod_{v \mid \infty} U_{v} K^{\times}\right) .
$$

Ce quotient est en bijection naturelle avec le groupe $\mathrm{Pic}^{+}\left(\mathscr{O}_{I}\right)$ des modules projectifs de rang 1 sur $O_{I}$ dans $K$, modulo l'équivalence au sens restreint. (Deux modules projectifs $M_{1}$ et $M_{2}$ sur $O_{I}$ sont dits équivalents au sens restreint s'il existe un élément totalement positif $k \in K_{+}^{\times}$tel que $M_{2}=k M_{1}$.) On associe en effet à tout $\alpha \in G_{I}$ un $O_{I}$-module $M \subset K$ en posant

$$
M^{\alpha}:=\alpha \hat{O}_{I} \cap K .
$$


L'application $\alpha \mapsto M^{\alpha}$ fournit une bijection naturelle :

$$
G_{I} \stackrel{\sim}{\longrightarrow}\left\{\begin{array}{c}
\text { classes d'équivalence } \\
\text { au sens restreint de } \\
O_{I} \text {-modules projectifs }
\end{array}\right\} .
$$

Soit $V:=\mathbb{O}_{I, 1}^{\times} \subset \mathbb{O}_{I}^{\times}$le groupe des unités de $\mathscr{O}_{I}$ de norme 1 sur $\mathbb{Q}$. Il laisse stable le module $M$. On a la suite exacte

$$
0 \longrightarrow V_{1} \longrightarrow V \longrightarrow \mathrm{O}_{F, 1}^{\times},
$$

où $V_{1}$ désigne le sous-groupe des unités de $V$ de norme relative 1 sur $F$. On note alors $\tilde{V}$ le sous-groupe de $V$ engendré par $V_{1}$ et $\mathcal{O}_{F}^{\times}$, et l'on pose

$$
\delta_{I}:=[V: \tilde{V}] .
$$

Cet indice est un diviseur de $2^{n}$. On définit finalement la fonction $L(M, s)$ associée à un $\mathrm{O}_{I}$-module projectif $M$ en posant

$$
L(M, s):=(\mathrm{N} M)^{s} \delta_{I} \sum_{x \in M / V}^{\prime} \operatorname{signe}\left(N_{K / \mathbb{Q}}(x)\right)\left|\mathrm{N}_{K / \mathbb{Q}}(x)\right|^{-s} .
$$

Remarque 1.3. (a) Parce que signe $\left(N_{K / \mathbb{Q}}(x)\right)=1$ quand $K$ est quadratique imaginaire, il en résulte que la définition (21) généralise l'équation (3) de l'introduction.

(b) Quand $K$ est une extension ATR de $F$ ayant $v_{1}$ pour unique place complexe, la fonction $L(M, s)$ est un multiple rationnel non-nul de la somme de fonctions $L$ partielles de Hurwitz de l'introduction. Plus précisément, si $a$ est un générateur de $\mathfrak{O}_{K} /\left(\mathscr{O}_{F}, I\right)$ en tant que $\left(\mathscr{O}_{F} / I\right)$-module, et que $M_{a}$ désigne le $\mathscr{O}_{I}$-module projectif

$$
M_{a}:=\left\{x \in \mathcal{O}_{K} \text { tel qu'il existe } r \in \mathscr{O}_{F} \text { avec } x \equiv r a \quad(\bmod I)\right\},
$$

alors

$$
L\left(M_{a}, s\right)=\delta_{I} \sum_{r \in \mathscr{O}_{F} / I \mathscr{O}_{F}} L(r a, I, s) .
$$

On vérifie que la fonction $L(M, s)$ ne dépend que de la classe d'équivalence de $M$ au sens restreint, puisque $L(\lambda M, s)=\operatorname{signe}\left(N_{K / \mathbb{Q}}(\lambda)\right) L(M, s)$.

Dans les sections 2 et 3 qui suivent, nous allons exprimer les valeurs spéciales $L(M, 0)$ quand $K$ est totalement réel, et les dérivées $L^{\prime}(M, 0)$ quand $K$ est ATR, en fonction de périodes appropriées de la forme différentielle $\omega_{\text {Eis }}$.

\section{Extensions quadratiques totalement réelles et valeurs de fonctions $L$}

On supposera dans cette section que l'extension quadratique $K$ de $F$ est totalement réelle. On veut rappeler un théorème qui apparaît dans la thèse du premier auteur et qui donne une formule explicite pour $L(M, 0)$ dans ce cas. 
L'hypothèse que $F$ a nombre de classes 1 au sens restreint implique que le module $M$ est libre de rang 2 comme module sur $\mathscr{O}_{F}$, et qu'il existe une $\mathscr{O}_{F}$-base $\left(\omega_{1}, \omega_{2}\right)$ de $M$. On suppose que cette base est choisie de sorte que $\left(1, \omega_{2} / \omega_{1}\right)$ soit orientée positivement. L'invariant $\tau:=\omega_{2} / \omega_{1}$ appartient à $K^{\times}$, et il ne dépend que de la classe d'équivalence de $M$ au sens restreint, à l'action de $\Gamma$ près. Le groupe $\Gamma_{\tau} \subset \Gamma$ formé des matrices qui fixent $\tau$ est un groupe de rang $n$ (modulo torsion), que l'application

$$
A=\left(\begin{array}{ll}
a & b \\
c & d
\end{array}\right) \mapsto c \tau+d
$$

identifie avec le sous-groupe $V_{1}$ des unités de $V$ de norme relative 1 sur $F$. Pour chaque $1 \leq j \leq n$, on pose

$$
\left(\tau_{j}, \tau_{j}^{\prime}\right):=v_{j}(\tau) \in \mathbb{R} \times \mathbb{R},
$$

et l'on appelle $\Upsilon_{j}$ la géodésique hyperbolique sur $\mathscr{H}_{j}$ joignant $\tau_{j}^{\prime}$ à $\tau_{j}$, orientée dans le sens allant de $\tau_{j}^{\prime}$ à $\tau_{j}$. Le produit

$$
R_{\tau}=\Upsilon_{1} \times \Upsilon_{2} \times \cdots \times \Upsilon_{n} \subset \mathscr{H}^{n}
$$

est un espace contractile homéomorphe à $\mathbb{R}^{n}$. On le munit de l'orientation naturelle héritée des $\Upsilon_{j}$. Le groupe $\Gamma_{\tau}$ opère sur $R_{\tau}$ par transformations de Möbius, et le quotient $\Gamma_{\tau} \backslash R_{\tau}$ est compact, isomorphe à un tore réel de dimension $n$. Soit $\Delta_{\tau}$ un domaine fondamental pour l'action de $\Gamma_{\tau}$ sur $R_{\tau}$. On identifie $\Delta_{\tau}$ avec son image dans $X$, qui est un cycle fermé dans ce quotient.

Théorème 2.1. Pour tout $\mathbb{O}_{I}$-module projectif $M$ dans $K$, on $a$ :

$$
(-2)^{n} \int_{\Delta_{\tau}} \omega_{\text {Eis }}=(2 i \pi)^{n} L(M, 0) .
$$

Démonstration. C'est une conséquence du corollaire 7.2 qui est démontré dans la dernière partie de cet article. Puisque $K$ est une extension quadratique totalement réelle de $F$, on choisit $r=n \geq 2$ et $c=0$ dans la formule (45). Elle s'écrit alors

$$
L(M, 0)=\frac{i^{n}}{\pi^{n}} \int_{\Delta_{\tau}} \frac{\partial^{n} \tilde{h}\left(z_{1}, \ldots, z_{n}\right)}{\partial z_{1} \cdots \partial z_{n}} d z_{1} \cdots d z_{n} .
$$

D'après l'identité (13), on en déduit que

$$
L(M, 0)=\frac{i^{n}}{\pi^{n}} \int_{\Delta_{\tau}} \frac{(2 i \pi)^{n}}{\sqrt{d_{F}}} \omega_{E_{2}} .
$$

La formule (23) en résulte immédiatement lorsque $n>2$ vu la définition (8) de $\omega_{\text {Eis }}$. Cette formule reste encore valable pour $n=2$ puisque les intégrales des formes $d z_{1} \wedge d \bar{z}_{1} / y_{1}^{2}$ et $d z_{2} \wedge d \bar{z}_{2} / y_{2}^{2}$ sur le cycle $\Delta_{\tau}$ sont nulles. 
Corollaire 2.2. Pour tout réseau $M$ dans $K$, les valeurs spéciales $L(M, 0)$ sont rationnelles. Plus précisément, il existe une constante entière $e_{F}$, ne dépendant que du corps $F$ et pas de l'extension $K$ ni de $M$, telle que $e_{F} L(M, 0) \in \mathbb{Z}$.

Démonstration. Cela résulte de ce que les périodes de $\omega_{\text {Eis }}$, d'après la proposition 1.2 , appartiennent à un réseau $\Lambda_{\text {Eis }} \subset(2 i \pi)^{n} \mathbb{Q}$ qui ne dépend que du corps $F$.

\section{Extensions quadratiques ATR et dérivées de fonctions $L$}

On suppose dans cette section que l'extension quadratique $K$ de $F$ est ATR, et que $v_{1}$ se prolonge en une place complexe de $K$. On veut donner dans ce cas une formule explicite pour $L^{\prime}(M, 0)$, lorsque $M$ est un $\mathscr{O}_{I}$-module projectif dans $K$.

Comme dans la section précédente, on pose $\tau:=\omega_{2} / \omega_{1}$, où $\left(\omega_{1}, \omega_{2}\right)$ est une $\mathrm{O}_{F}$-base positive de $M$, choisie de sorte que $(1, \tau)$ soit orientée positivement. On pose ensuite

$$
\begin{cases}\tau_{1}:=v_{1}(\tau) & \in \mathscr{H}_{1} \\ \left(\tau_{j}, \tau_{j}^{\prime}\right):=v_{j}(\tau) & \in \mathbb{R} \times \mathbb{R}, \text { pour } j=2, \ldots, n\end{cases}
$$

Le nombre complexe $\tau_{1}$ appartient alors à $\mathscr{H}_{1}$. Pour chaque $2 \leq j \leq n$, on appelle $\Upsilon_{j}$ la géodésique hyperbolique de $\mathscr{H}_{j}$ joignant $\tau_{j}$ à $\tau_{j}^{\prime}$, orientée dans le sens allant de $\tau_{j}$ à $\tau_{j}^{\prime}$. Le produit

$$
R_{\tau}=\left\{\tau_{1}\right\} \times \Upsilon_{2} \times \cdots \times \Upsilon_{n} \subset \mathscr{H}^{n}
$$

est un espace contractile homéomorphe à $\mathbb{R}^{n-1}$, que l'on munit de l'orientation naturelle héritée des $\Upsilon_{j}$. Le stabilisateur $\Gamma_{\tau}$ de $\tau$ dans $\Gamma$ est un groupe de rang $n-1$ modulo torsion, que l'on peut identifier avec le sous-groupe d'unités relatives $V_{1}$ introduit précédemment. Il opère sur $R_{\tau}$ par transformations de Möbius, et le quotient $\Gamma_{\tau} \backslash R_{\tau}$ est compact, isomorphe à un tore réel de dimension $(n-1)$. Soit $\Delta_{\tau}$ un domaine fondamental pour l'action de $\Gamma_{\tau}$ sur $R_{\tau}$. On identifie $\Delta_{\tau}$ avec son image dans $X$, qui est un cycle fermé de dimension $n-1$ dans ce quotient.

Lemme 3.1. La classe de $\Delta_{\tau}$ dans $H_{n-1}(X, \mathbb{Z})$ est de torsion. En particulier, il existe une $n$-chaîne différentiable $C_{\tau}$ à coefficients dans $\mathbb{Q}$ telle que

$$
\partial C_{\tau}=\Delta_{\tau} .
$$

Démonstration. Le sous-groupe de torsion de $H_{n-1}(X, \mathbb{Z})$ s'identifie avec le noyau de l'application naturelle $H_{n-1}(X, \mathbb{Z}) \rightarrow H_{n-1}(X, \mathbb{Q})$. Lorsque $n$ est pair, le lemme 3.1 résulte de ce que $H_{n-1}(X, \mathbb{Q})=0$ [Freitag 1990, chap. III]. De même, lorsque $n=2 m+1$ est impair, le groupe $H^{n-1}(X, \mathbb{C})$ est engendré par les classes des $\left(\begin{array}{l}n \\ m\end{array}\right)$ 
formes différentielles du type

$$
\eta_{S}:=\bigwedge_{j \in S} \frac{d z_{j} \wedge d \bar{z}_{j}}{y_{j}^{2}}, \quad S \subset\{1, \ldots, n\}, \quad \# S=m .
$$

Or on voit que les restrictions de ces classes sur $\Delta_{\tau}$ (et même sur les régions $R_{\tau}$ ) sont nulles, puisque la projection de $R_{\tau}$ sur chaque facteur $\mathscr{H}_{j}$ est de dimension réelle 0 ou 1 . On en déduit par la dualité de Poincaré que l'image de $\Delta_{\tau}$ dans $H_{n-1}(X, \mathbb{C})$ est nulle.

On introduit $\omega_{\text {Eis }}+=\frac{1}{2}\left(\mathrm{Id}+T_{v_{1}}^{*}\right) \omega_{\text {Eis }}$ la projection de la forme différentielle $\omega_{\text {Eis }}$ sur l'espace propre de $T_{v_{1}}^{*}$ associé à la valeur propre +1 , autrement dit la «partie réelle pour la place $v_{1} \gg$ de la forme $\omega_{\text {Eis }}$.

Théorème 3.2. Soit $M$ un $\mathrm{O}_{I}$-module projectif associé à $\tau \in K$. L'intégrale de $\omega_{\mathrm{Eis}}^{+}$ sur $C_{\tau}$ ne dépend pas du choix de $C_{\tau}$ vérifiant (26), et l'on a

$$
(-2)^{n-1} \int_{C_{\tau}} \omega_{\text {Eis }}^{+}=(2 i \pi)^{n-1} L^{\prime}(M, 0) .
$$

Démonstration. C'est encore une conséquence du corollaire 7.2 qui est démontré dans la dernière partie de cet article. La première assertion découle du fait que $\omega_{\text {Eis }}^{+}$ est exacte : le lemme 1.1 montre que $\omega_{\text {Eis }}^{+}=d \eta / 2$. Le calcul se poursuit en utilisant le théorème de Stokes pour obtenir

$$
\int_{C_{\tau}} \omega_{\text {Eis }}^{+}=\int_{\Delta_{\tau}} \frac{\eta}{2}
$$

Supposons tout d'abord que $n>2$, de sorte que $\eta$ est la (n-1)-forme holomorphe sur $X$ donnée par la formule (15). Comme $K$ est une extension ATR, on choisit ici $r=n-1 \geq 2$ et $c=1$ dans le corollaire 7.2.i. La formule correspondante s'écrit

$$
L^{\prime}(M, 0)=\frac{i^{n-1}}{2 \pi^{n-1}} \int_{\Delta_{\tau}} \eta,
$$

ce qui permet de conclure.

Il reste à traiter le cas où $n=2$ en faisant cette fois appel au corollaire 7.2.ii. On choisit $r=1$ et $c=1$ dans la formule (46) qui s'écrit

$$
L^{\prime}(M, 0)=\frac{i}{2 \pi} \int_{\Delta_{\tau}}\left(\frac{\partial \tilde{h}}{\partial z_{2}}-\frac{4 R_{F}}{z_{2}-\bar{z}_{2}}\right) d z_{2} .
$$

Au vu de la définition (17) de la forme $\eta$ pour $n=2$, cette égalité se réduit à

$$
\int_{\Delta_{\tau}}-\eta=(2 i \pi) L^{\prime}(M, 0) \text {. }
$$

La formule de Stokes permet de nouveau de conclure à la formule souhaitée. 


\section{Application d'Abel-Jacobi et unités de Stark}

Quand on combine le théorème 3.2 avec la conjecture 1 de Stark, on obtient la formule conjecturale suivante pour le logarithme du module de l'unité de Stark :

$$
e_{I}(-2)^{n-1} \int_{C_{\tau}} \omega_{\text {Eis }}+=2 \delta_{I}(2 i \pi)^{n-1} \log \left|\tilde{v}_{1}\left(u_{\tau}\right)\right|,
$$

où $e_{I}$ désigne l'ordre du groupe des racines de l'unité dans $H_{I}$, et l'entier $\delta_{I}$ est défini dans l'équation (20). Pour relever l'invariant réel $\log \left|\tilde{v}_{1}\left(u_{\tau}\right)\right|=\operatorname{Re} \log \tilde{v}_{1}\left(u_{\tau}\right)$ en un invariant complexe bien défini modulo $2 i \pi \mathbb{Z}$, il suffira de remplacer dans la formule (30) la différentielle exacte $\omega_{\text {Eis }}^{+}$par la forme différentielle $\omega_{\text {Eis }}$.

Pour tout $m \geq 0$, on désigne par $C_{m}(X)$ le groupe engendré par les combinaisons linéaires formelles à coefficients dans $\mathbb{Z}$ des chaînes différentiables de dimension réelle $m$ sur $X$, et l'on désigne par $C_{m}^{0}(X)$ et $C_{m}^{00}(X)$ les sous-groupes engendrés par les cycles différentiables fermés et homologues à zéro respectivement :

$$
\begin{aligned}
C_{m}^{0}(X) & :=\left\{\Delta \in C_{m}(X) \text { tel que } \partial \Delta=0\right\} . \\
C_{m}^{00}(X) & :=\left\{\Delta \in C_{m}(X) \text { tel qu'il existe } C \in C_{m+1}(X) \text { avec } \partial C=\Delta\right\} .
\end{aligned}
$$

On pose aussi

$$
\tilde{C}_{m}^{00}(X):=\left\{\Delta \in C_{m}(X) \text { tel qu'il existe } C \in C_{m+1}(X) \otimes \mathbb{Q} \text { avec } \partial C=\Delta\right\} .
$$

On sait que $C_{n-1}^{0}(X) / C_{n-1}^{00}(X)=H_{n-1}(X, \mathbb{Z})$ est un groupe de type fini, dont le sous-groupe de torsion s'identifie avec $\tilde{C}_{n-1}^{00}(X) / C_{n-1}^{00}(X)$. Soit $n_{F}$ l'exposant de ce groupe fini, et soit

$$
\Lambda_{\text {Eis }}^{\prime}:=\frac{1}{n_{F}} \Lambda_{\text {Eis }} .
$$

On peut définir à partir de la forme différentielle $\omega_{\text {Eis }}$ une «application d'AbelJacobi»

$$
\Phi_{\text {Eis }}: C_{n-1}^{00}(X) \rightarrow \mathbb{C} / \Lambda_{\text {Eis }}
$$

en posant

$$
\Phi_{\text {Eis }}(\Delta)=\int_{\partial C=\Delta} \omega_{\text {Eis }}\left(\bmod \Lambda_{\text {Eis }}\right),
$$

l'intégrale étant prise sur n'importe quelle $n$-chaîne différentiable $C$ sur $X$ tel que $\partial C=\Delta$. Cette application $\Phi_{\text {Eis }}$ est bien définie modulo le réseau des périodes $\Lambda_{\text {Eis }}$ en vertu de la proposition 1.2. Quitte à remplacer le réseau $\Lambda_{\text {Eis }}$ par $\Lambda_{\text {Eis }}^{\prime}$, on peut étendre $\Phi_{\text {Eis }}$ au groupe $\tilde{C}_{n-1}^{00}(X)$ tout entier, en posant

$$
\Phi_{\text {Eis }}(\Delta)=\frac{1}{n_{F}} \int_{\partial C=n_{F} \Delta} \omega_{\text {Eis }}\left(\bmod \Lambda_{\text {Eis }}^{\prime}\right),
$$


l'intégrale étant prise sur n'importe quelle $n$-chaîne différentiable $C$ sur $X$ tel que $\partial C=n_{F} \Delta$. On pose ensuite

$$
J_{\tau}:=e_{I}(-2)^{n-1} \Phi_{\text {Eis }}\left(\Delta_{\tau}\right),
$$

élément de $\mathbb{C} / \Lambda_{\text {Eis }}^{\prime}$.

Soit $\Lambda_{\text {Eis }}^{\prime \prime}$ le réseau de $(2 i \pi)^{n} \mathbb{R}$ engendré par $\Lambda_{\text {Eis }}^{\prime}$ et $(2 i \pi)^{n} \mathbb{Z}$. On fixe une place $\tilde{v}_{1}$ de $H_{I}$ au-dessus de la place $v_{1}$ de $K$. Nous sommes maintenant en mesure d'énoncer la conjecture principale de cet article.

Conjecture 4.1. Pour tout $\widehat{O}_{I}$-module $M$ associé à $\tau \in K$, il existe une unité $u_{\tau} \in$ $\mathrm{O}_{H_{I}}^{\times}$telle que

$$
J_{\tau}=2 \delta_{I}(2 i \pi)^{n-1} \log \left(\tilde{v}_{1}\left(u_{\tau}\right)\right) \quad\left(\bmod \Lambda_{\text {Eis }}^{\prime \prime}\right) .
$$

De plus, pour tout $2 \leq j \leq n$, l'image de $u_{\tau}$ par n'importe quel plongement complexe au-dessus de $v_{j}$ est de module 1 . Pour tout $\alpha \in G_{I}$, on a $u_{\tau^{\alpha}}=\operatorname{rec}(\alpha)^{-1} u_{\tau}$, où $\tau^{\alpha}$ désigne l'invariant associé au module $M^{\alpha}$.

\section{Algorithmes}

L'invariant $J_{\tau}$ et l'application d'Abel-Jacobi $\Phi_{\text {Eis }}$ ont l'inconvénient de ne pas être faciles à calculer numériquement a priori. Le but de la présente section est de décrire un algorithme pour le calcul de $\Phi_{\text {Eis }}$ dans le cas le plus simple où $n=2$.

La première étape consiste à décrire la classe de cohomologie de $\omega_{\text {Eis }}$ en termes de cohomologie du groupe $\Gamma$.

On rappelle le dictionnaire bien connu entre la cohomologie de De Rham de $X$ et la cohomologie de $\Gamma$. Si $P, Q, R$ sont des points de $\mathscr{H}_{1} \times \mathscr{H}_{2}=\mathscr{H}^{2}$, on appelle $\Delta(P, Q, R)$ n'importe quelle 2-chaîne différentiable dont la frontière est égale au triangle géodésique de sommets $P, Q$ et $R$. On munit cette région de l'orientation standard, selon les définitions usuelles de l'homologie singulière. On pose aussi, pour $P=\left(z_{1}, z_{2}\right) \in \mathscr{H}^{2}$ et $A, B \in \Gamma$,

$$
\Delta_{P}(A, B):=\Delta(P, A P, A B P) .
$$

On associe à $\omega_{\text {Eis }}$ (plus précisément : à sa classe de cohomologie) un 2-cocycle

$$
\kappa_{P} \in Z^{2}(\Gamma, \mathbb{C})
$$

par la règle

$$
\kappa_{P}(A, B):=\int_{\Delta_{P}(A, B)} \omega_{\mathrm{Eis}} .
$$

Un calcul direct montre que $\kappa_{P}$ satisfait la relation de 2-cocycle : $d \kappa_{P}=0$, et que son image dans $H^{2}(\Gamma, \mathbb{C})$ ne dépend pas du choix du point base $P$.

On rappelle le réseau $\Lambda_{\text {Eis }} \subset \mathbb{C}$ des périodes de $\omega_{\text {Eis }}$ et l'on note $\bar{\kappa}_{P}$ l'image de $\kappa_{P}$ dans $Z^{2}\left(\Gamma, \mathbb{C} / \Lambda_{\text {Eis }}^{\prime}\right)$. 
Lemme 5.1. La classe de $\bar{\kappa}_{P}$ dans $H^{2}\left(\Gamma, \mathbb{C} / \Lambda_{\text {Eis }}^{\prime}\right)$ est nulle.

Démonstration. Pour tout $A \in \Gamma$, on appelle $S_{P}(A)$ l'image dans $X$ du chemin géodésique sur $\mathscr{H}^{2}$ allant de $P$ à $A P$. Comme $S_{P}(A)$ est un 1 -cycle fermé sur $X$ et que $H_{1}(X, \mathbb{Q})=0$, il existe une 2-chaîne différentiable sur $X$ à coefficients entiers, que l'on appellera $D_{P}(A)$, telle que

$$
\partial D_{P}(A)=n_{F} S_{P}(A) .
$$

La région $D_{P}(A)$ est déterminée par cette équation modulo les 2-cycles fermés, et par conséquent l'élément de $\mathbb{C} / \Lambda_{\text {Eis }}^{\prime}$ défini par

$$
\rho_{P}(A):=\frac{1}{n_{F}} \int_{D_{P}(A)} \omega_{\text {Eis }} \quad\left(\bmod \Lambda_{\text {Eis }}^{\prime}\right)
$$

ne dépend pas du choix de $D_{P}(A)$ satisfaisant (32). On vérifie ensuite par un calcul direct que

$$
d \rho_{P}(A, B)=\kappa_{P}(A, B) \quad\left(\bmod \Lambda_{\text {Eis }}^{\prime}\right) .
$$

Le lemme 5.1 permet de définir une 1-chaîne $\rho_{P}$ en choisissant une solution de l'équation

$$
d \rho_{P}=\kappa_{P} \quad\left(\bmod \Lambda_{\text {Eis }}^{\prime}\right) .
$$

Soit $K$ un corps ATR et soit $\tau \in K$ un élément provenant d'une base positive d'un réseau $M \subset K$. Parce que $n=2$, le groupe $\Gamma_{\tau}$ est de rang un modulo la torsion. On se donne un générateur $\gamma_{\tau}$ de $\Gamma_{\tau}$ modulo torsion, choisi de sorte que pour tout point $z_{2}$ de la géodésique $\Upsilon_{2}$, le chemin allant de $z_{2}$ à $\gamma_{\tau} z_{2}$ soit orienté dans le sens positif. On choisit le point base $P \in \mathscr{H}_{1} \times \mathscr{H}_{2}$ de manière à ce que sa première composante soit égale à $\tau_{1}=v_{1}(\tau)$.

La proposition suivante, qui résulte directement de la formule pour $\rho_{P}$ de l'équation (33), permet de calculer l'invariant numérique $\Phi_{\text {Eis }}\left(\Delta_{\tau}\right)$ en termes de cohomologie des groupes — du moins, en admettant que l'on sache résoudre (34).

\section{Proposition 5.2. $\quad \Phi_{\text {Eis }}\left(\Delta_{\tau}\right)=\rho_{P}\left(\gamma_{\tau}\right)$.}

La définition du 2-cocycle $\kappa_{P}$ exige d'intégrer $\omega_{\text {Eis }}$ sur des régions de type $\Delta_{P}(A, B)$ peu commodes à paramétrer. Dans les calculs numériques, il est donc utile de remplacer ce cocycle par un représentant de la même classe de cohomologie qui ne fait intervenir que des régions rectangulaires de la forme $L_{1} \times L_{2} \subset \mathscr{H}_{1} \times \mathscr{H}_{2}$ (avec $L_{1}$ et $L_{2}$ de dimension 1, bien entendu). Les intégrales de $\omega_{\text {Eis }}$ sur de telles régions s'expriment au moyen d'intégrales itérées, et sont donc plus faciles à calculer numériquement. (On se sert pour cela du développement de Fourier de $\omega_{\text {Eis }}$.)

Si $u, v$ appartiennent à $\mathscr{H}$, soit $\Upsilon[u, v] \subset \mathscr{H}$ le segment géodésique joignant le point $u$ au point $v$. On pose

$$
\square_{P}(A, B)=\Upsilon\left[z_{1}, A_{1} z_{1}\right] \times \Upsilon\left[A_{2} z_{2}, A_{2} B_{2} z_{2}\right],
$$


et l'on définit un nouveau cocycle $\kappa_{P}^{\square} \in Z^{2}(\Gamma, \mathbb{C})$ par la règle

$$
\kappa_{P}^{\square}(A, B)=\int_{\square_{P}(A, B)} \omega_{\text {Eis }} .
$$

Il est nécessaire de modifier légèrement $\kappa_{P}^{\square}$ pour qu'il représente la même classe de cohomologie que $\kappa_{P}$. On dispose pour cela d'un 2-cocycle classique $\operatorname{sur} \mathbf{S L}_{2}(\mathbb{R})$ appelé cocycle d'aire, dont on rappelle la définition : étant données deux matrices $M=\left(\begin{array}{ll}* & * \\ c & d\end{array}\right)$ et $N=\left(\begin{array}{cc}* & * \\ c^{\prime} & d^{\prime}\end{array}\right)$ de $\mathbf{S L}_{2}(\mathbb{R})$, avec leur produit $M N=\left(\begin{array}{cc}* & * \\ c^{\prime \prime} & d^{\prime \prime}\end{array}\right)$, la formule

$$
\operatorname{aire}(M, N):=-\operatorname{signe}\left(c c^{\prime} c^{\prime \prime}\right)
$$

(où signe $(x)=x /|x|$ si $x \neq 0$, et 0 sinon) définit un 2-cocycle à valeurs entières. Par composition avec les plongements de $\Gamma$ dans $\mathbf{S L}_{2}(\mathbb{R})$, on en déduit deux 2cocycles sur $\Gamma$ à valeurs dans $\mathbb{Z}$. On définit finalement le 2-cocycle $\tilde{\kappa}_{P}$ sur $\Gamma$ par la formule

$$
\begin{aligned}
\tilde{\kappa}_{P}(A, B): & =\kappa_{P}^{\square}(A, B)-i \pi R_{F} \operatorname{aire}\left(A_{1}, B_{1}\right)+i \pi R_{F} \operatorname{aire}\left(A_{2}, B_{2}\right) \\
& =\int_{z_{1}}^{A_{1} z_{1}} \int_{A_{2} z_{2}}^{A_{2} B_{2} z_{2}} \omega_{\text {Eis }}-i \pi R_{F} \operatorname{aire}\left(A_{1}, B_{1}\right)+i \pi R_{F} \operatorname{aire}\left(A_{2}, B_{2}\right) .
\end{aligned}
$$

Proposition 5.3. Les cocycles $\kappa_{P}$ et $\tilde{\kappa}_{P}$ représentent la même classe de cohomologie dans $H^{2}(\Gamma, \mathbb{C})$. Plus précisément, on a

$$
\kappa_{P}(A, B)-\tilde{\kappa}_{P}(A, B)=d \xi_{P}(A, B),
$$

où

$\xi_{P}(A)=-\int_{\Delta_{P}(A)} \omega_{\text {Eis }}, \quad$ avec $\Delta_{P}(A)=\Delta\left(\left(z_{1}, z_{2}\right),\left(z_{1}, A_{2} z_{2}\right),\left(A_{1} z_{1}, A_{2} z_{2}\right)\right)$.

Démonstration. On dit que deux 2-chaînes $Z_{1}$ et $Z_{2}$ sont homologues si leurs frontières sont égales, et on écrit dans ce cas $Z_{1} \sim Z_{2}$. Un calcul direct fournit la relation

$$
-\square_{P}(A, B)+\Delta_{P}(A, B)+\Delta_{P}(A)-\Delta_{P}(A B) \sim \Delta_{1}+\Delta_{2}-\Delta_{3},
$$

avec

$$
\left\{\begin{array}{l}
\Delta_{1}=\Delta\left(\left(A_{1} B_{1} z_{1}, A_{2} B_{2} z_{2}\right),\left(z_{1}, A_{2} B_{2} z_{2}\right),\left(A_{1} z_{1}, A_{2} B_{2} z_{2}\right)\right) \\
\Delta_{2}=\Delta\left(\left(z_{1}, z_{2}\right),\left(z_{1}, A_{2} z_{2}\right),\left(z_{1}, A_{2} B_{2} z_{2}\right)\right) \\
\Delta_{3}=\Delta\left(\left(A_{1} z_{1}, A_{2} z_{2}\right),\left(A_{1} z_{1}, A_{2} B_{2} z_{2}\right),\left(A_{1} B_{1} z_{1}, A_{2} B_{2} z_{2}\right)\right) .
\end{array}\right.
$$

Par $A$-invariance, on observe que $\Delta_{3}=\Delta_{P}(B)$ dans $X$. En outre, l'intégrale de $\omega_{E_{2}}$ sur $\Delta_{1}$ et $\Delta_{2}$ est nulle, et par conséquent 


$$
\int_{\Delta_{1}+\Delta_{2}} \omega_{\text {Eis }}=\frac{R_{F}}{2} \int_{\Delta_{1}} \frac{d z_{1} \wedge d \bar{z}_{1}}{y_{1}^{2}}-\frac{R_{F}}{2} \int_{\Delta_{2}} \frac{d z_{2} \wedge d \bar{z}_{2}}{y_{2}^{2}} .
$$

Ces dernières intégrales se calculent élémentairement : on constate d'abord qu'elles ne dépendent pas du point base $P=\left(z_{1}, z_{2}\right)$, et que $d z_{j} \wedge d \bar{z}_{j}=-2 i d x_{j} \wedge d y_{j}$. Or l'intégrale

$$
\int_{\Delta_{j}} \frac{d x_{j} \wedge d y_{j}}{y_{j}^{2}}
$$

n'est rien d'autre que l'aire, dans le disque de Poincaré, du triangle idéal orienté de sommets $\infty, A_{j} \infty$ et $A_{j} B_{j} \infty$. D'après [Kirby et Melvin 1994] formule 1.2, il en résulte que

$$
\int_{\Delta_{1}} \frac{d z_{1} \wedge d \bar{z}_{1}}{y_{1}^{2}}=-2 i \pi \operatorname{aire}\left(A_{1}, B_{1}\right) \text { et } \int_{\Delta_{2}} \frac{d z_{2} \wedge d \bar{z}_{2}}{y_{2}^{2}}=-2 i \pi \operatorname{aire}\left(A_{2}, B_{2}\right) .
$$

On conclut alors de (36) que

$$
\kappa_{P}(A, B)=\kappa_{P}^{\square}(A, B)+d \xi_{P}(A, B)-i \pi R_{F} \text { aire }\left(A_{1}, B_{1}\right)+i \pi R_{F} \text { aire }\left(A_{2}, B_{2}\right),
$$

d'où la proposition.

Corollaire 5.4. Soit $\tilde{\rho}_{P}$ une solution de l'équation

$$
d \tilde{\rho}_{P}=\tilde{\kappa}_{P} \quad\left(\bmod \Lambda_{\text {Eis }}^{\prime}\right) .
$$

Alors on a $\Phi_{\text {Eis }}\left(\Delta_{\tau}\right)=\tilde{\rho}_{P}\left(\gamma_{\tau}\right)$.

Démonstration. La proposition 5.3 montre que l'on peut choisir

$$
\tilde{\rho}_{P}(A)=\rho_{P}(A)-\xi_{P}(A) \quad\left(\bmod \Lambda_{\text {Eis }}^{\prime}\right),
$$

où la 1-cochaîne $\xi_{P}$ est définie dans l'énoncé de cette proposition. Comme la région $\Delta_{P}\left(\gamma_{\tau}\right)$ qui intervient dans la formule pour $\xi_{P}\left(\gamma_{\tau}\right)$ est contenue dans le domaine $\left\{\tau_{1}\right\} \times \Upsilon\left[z_{2}, \gamma_{\tau} z_{2}\right]$, on a

$$
\xi_{P}\left(\gamma_{\tau}\right)=0
$$

Le corollaire en résulte.

Remarque 5.5. Dans le présent article, le cocycle $\tilde{\kappa}_{P}$ n'intervient que dans les algorithmes pour calculer $J_{\tau}$ numériquement. Signalons tout de même que la proposition 5.3 et le corollaire 5.4 sont d'un intérêt plus que pratique. Dans le contexte partiellement $p$-adique étudié dans [Darmon 2001] et [Darmon et Dasgupta 2006] où l'on est amené à travailler avec des formes modulaires sur $\mathscr{H}_{p} \times \mathscr{H}$, on ignore comment donner un sens aux régions de la forme $\Delta_{P}(A, B)$, ou au cocycle $\kappa_{P}$. Par contre, on sait définir ce qui doit jouer le rôle des intégrales «itérées» de formes modulaires (cuspidales ou Eisenstein) sur des régions «rectangulaires» de la forme $\square_{P}(A, B)$. Cela permet de définir un avatar $p$-adique de $\tilde{\kappa}_{P}$, et par conséquent des versions $p$-adiques des invariants $J_{\tau}$ du présent article. 
Il reste finalement à calculer une solution de l'équation (34) ou (37). Le procédé étant le même qu'il s'agisse de $\rho_{P}$ ou de $\tilde{\rho}_{P}$, on se bornera au cas de $\rho_{P}$ pour alléger les notations.

L'algorithme que nous proposons pour calculer $\rho_{P}(\gamma)$, pour $\gamma$ n'importe quel élément de $\Gamma$, se base sur l'observation suivante : lorsque $\gamma=h k h^{-1} k^{-1}$ est un commutateur dans $\Gamma$, la formule (34) permet d'exprimer $\rho_{P}(\gamma)$ directement en fonction de $\kappa_{P}$. En effet, l'identité facile $\rho_{P}(\mathrm{Id})=0$ assure que

$$
\rho_{P}(h)+\rho_{P}\left(h^{-1}\right)=\kappa_{P}\left(h, h^{-1}\right) .
$$

En reportant, on en conclut que

$$
\begin{aligned}
& \rho_{P}(\gamma)=-\kappa_{P}\left(h, k h^{-1} k^{-1}\right)-\kappa_{P}\left(k, h^{-1} k^{-1}\right)-\kappa_{P}\left(h^{-1}, k^{-1}\right) \\
& +\kappa_{P}\left(h, h^{-1}\right)+\kappa_{P}\left(k, k^{-1}\right) \quad\left(\bmod \Lambda_{\text {Eis }}^{\prime}\right) .
\end{aligned}
$$

Cette formule, avec $\rho_{P}$ et $\kappa_{P}$ remplacés par $\tilde{\rho}_{P}$ et $\tilde{\kappa}_{P}$ respectivement, donne un accès numérique à $\tilde{\rho}_{P}\left(h k h^{-1} k^{-1}\right)$ puisque les nombres complexes $\tilde{\kappa}_{P}\left(g, g^{\prime}\right)$ se calculent grâce au développement en série de Fourier de la série d'Eisenstein.

Enfin, l'abélianisé $\Gamma_{\mathrm{ab}}$ de $\Gamma$ est fini (voir [Darmon et Logan 2003, prop. 1.3]). Son ordre divise $4 N_{F / \mathbb{Q}}\left(\epsilon^{2}-1\right)$, où $\epsilon$ désigne l'unité fondamentale de $F$. Pour calculer $\rho_{P}(\gamma)$ pour une matrice $\gamma$ de $\Gamma$, il suffit donc de décomposer $\gamma^{\left|\Gamma_{a b}\right|}$ en un produit de commutateurs.

Sous l'hypothèse que $F$ est de nombre de classes 1 , on peut procéder comme suit. L'anneau des entiers $\hat{O}_{F}$ est euclidien en $k$-étapes pour la norme selon la terminologie de [Cooke 1976, théorème 1]. Par conséquent, le groupe modulaire de Hilbert $\Gamma$ est engendré par les matrices élémentaires de type suivant :

$$
S=\left(\begin{array}{rr}
0 & -1 \\
1 & 0
\end{array}\right), \quad T_{\theta}=\left(\begin{array}{ll}
1 & \theta \\
0 & 1
\end{array}\right), \quad U=\left(\begin{array}{cc}
\epsilon & 0 \\
0 & \epsilon^{-1}
\end{array}\right) .
$$

Ainsi, $\gamma^{\left|\Gamma_{a b}\right|}$ s'écrit comme un produit de matrices élémentaires grâce à l'algorithme d'Euclide dans $\widehat{O}_{F}$, puis comme un produit de commutateurs à l'aide des relations

$$
U T_{\theta} U^{-1} T_{\theta}^{-1}=T_{\theta\left(\epsilon^{2}-1\right)}, \quad S U S^{-1} U^{-1}=U^{2} .
$$

Remarque 5.6. Dans notre contexte «Eisenstein», on ne peut pas utiliser tel quel l'algorithme proposé dans [Darmon et Logan 2003, section 4]. En effet, les intégrales du type $\int^{\tau} \int_{c_{2}}^{c_{1}} \omega_{f}\left(\operatorname{avec} c_{1}, c_{2} \in \mathbf{P}^{1}(F)\right)$ n'ont de sens que si $f$ est une forme modulaire de Hilbert cuspidale. Notons cependant que cet algorithme et le nôtre reposent tous deux sur l'hypothèse que $O_{F}$ est un anneau euclidien. 


\section{Exemples numériques}

Dans cette partie, nous présentons quelques résultats expérimentaux obtenus grâce à l'algorithme précédent. Il s'agit, pour quelques cas d'extensions ATR $K / F$, de tester numériquement la conjecture 4.1 de cet article et d'exhiber le polynôme minimal de l'unité attendue.

6.1. Corps de base $\mathbb{Q}(\sqrt{5})$. On considère d'abord la situation où $F=\mathbb{Q}(\sqrt{5})$ et l'on note $\epsilon=\frac{1}{2}(1+\sqrt{5})$ son unité fondamentale de norme -1 . L'anneau des entiers $O_{F}=\mathbb{Z}[\epsilon]$ est euclidien pour la norme. On fixe les places archimédiennes $v_{1}$ et $v_{2}$ de $F$ de sorte que $\epsilon_{1}<0$ et $\epsilon_{2}>0$. On supposera dans cette section que $\Lambda_{\text {Eis }}^{\prime \prime}=\Lambda_{\text {Eis }}^{\prime}$, et l'on se donne un entier $m_{F}>0$ tel que $\Lambda_{\text {Eis }}^{\prime} \subset(2 i \pi)^{2} m_{F} \mathbb{Z}$. Les exemples ci-dessous laissent penser que $m_{\mathbb{Q}(\sqrt{5})}=15$ convient.

Nous étudions maintenant les invariants associés à différentes extensions quadratiques ATR $K$ de $F$ dans lesquelles la place $v_{1}$ devient complexe.

(a) Un exemple à groupe des classes $C_{4}$. On considère $K=F(\sqrt{21 \epsilon-11})$. C'est une extension ATR de $F$, dont le groupe des classes au sens restreint est cyclique d'ordre 4. Aux quatre classes distinctes $\mathscr{C}_{1}, \ldots, \mathscr{C}_{4}$ de $\mathscr{O}_{K}$ au sens restreint, on associe les éléments $\tau_{1}, \ldots, \tau_{4}$ de $K$ fixés par les matrices suivantes de $\Gamma$ :

$$
\begin{array}{ll}
\gamma_{1}=\left(\begin{array}{cc}
4 \epsilon+2 & -2 \epsilon-5 \\
-2 \epsilon-1 & 2 \epsilon+1
\end{array}\right), & \gamma_{2}=\left(\begin{array}{cc}
13 \epsilon+9 & 4 \epsilon+1 \\
-32 \epsilon-18-7 \epsilon-6
\end{array}\right), \\
\gamma_{3}=\left(\begin{array}{cc}
-47 \epsilon-17 & 9 \epsilon-6 \\
-520 \epsilon-308 & 53 \epsilon+20
\end{array}\right), & \gamma_{4}=\left(\begin{array}{cc}
165 \epsilon+79 & 5 \epsilon-2 \\
-8512 \epsilon-5160 & -159 \epsilon-76
\end{array}\right) .
\end{array}
$$

On calcule dans $\mathbb{C} / m_{F} \mathbb{Z}$ les invariants $\rho_{k}\left(\gamma_{k}\right):=\tilde{\rho}_{\tau_{k}}\left(\gamma_{k}\right) /(2 i \pi)^{2}$ associés. On trouve, avec une précision minimale de 50 décimales significatives :

$$
\begin{aligned}
& \rho_{1}\left(\gamma_{1}\right) \approx-0.3666666666 \ldots-0.27784944302 \ldots i \\
& \rho_{2}\left(\gamma_{2}\right) \approx 0.32623940638 \ldots ; \\
& \rho_{3}\left(\gamma_{3}\right) \approx 1.83333333333 \ldots+0.27784944302 \ldots i \\
& \rho_{4}\left(\gamma_{4}\right) \approx 17.8404272602 \ldots
\end{aligned}
$$

Sans connaître la constante $m_{F}$, on doit tester l'algébricité du nombre complexe bien défini

$$
u_{k}\left(m_{F}\right)=\exp \left(2 i \pi m_{F} \rho_{k}\left(\gamma_{k}\right)\right) \text {. }
$$

Cependant, dans la pratique, il semble qu'il existe toujours une racine $m_{F}$-ième de $u_{k}\left(m_{F}\right)$ qui appartienne au corps de définition de $u_{k}\left(m_{F}\right)$. Pour chaque valeur de $\rho_{k}\left(\gamma_{k}\right)$ dans la liste précédente, notons

$$
u_{k}(1)=\exp \left(2 i \pi \rho_{k}\left(\gamma_{k}\right)\right)
$$


Le nombre complexe $u_{k}(1)$ est bien défini seulement modulo les racines $m_{F}$-ièmes de l'unité. Quitte à modifier $u_{k}(1)$ par une racine de l'unité, on peut donc espérer tester avec succès son algébricité. Précisément, la commande Pari

$$
\operatorname{algdep}\left(u_{1}(1) e^{-\frac{4}{15} i \pi}, 16\right)
$$

suggère la relation algébrique suivante pour $u_{1}:=u_{1}(1) e^{-\frac{4}{15} i \pi}$ :

$$
Q_{1}(x):=x^{8}+4 x^{7}-10 x^{6}+x^{5}+9 x^{4}+x^{3}-10 x^{2}+4 x+1 .
$$

On en conclut que le nombre complexe $u_{1}$ coïncide sur 50 décimales avec la racine $-5.7303 \ldots$ du polynôme $Q_{1}$. Il en va de même pour les trois autres invariants : $u_{2}:=u_{2}(1) e^{\frac{23}{15} i \pi}$ coïncide avec la racine $0.834403847893 \ldots+0.5511535345 \ldots i$ de $Q_{1}$.

$u_{3}:=u_{3}(1) e^{\frac{20}{15} i \pi}$ coïncide avec la racine $-0.17450889906 \cdots=1 / u_{1}$. $u_{4}:=u_{4}(1) e^{\frac{2}{15} i \pi}$ coïncide avec la racine $0.834403847893 \ldots-0.5511535345 \ldots i$.

On vérifie a posteriori que $Q_{1}$ est effectivement le polynôme minimal d'une unité du corps de classes de Hilbert (au sens restreint) de $K$.

(b) Un exemple à groupe des classes $C_{6}$. On considère maintenant

$$
K=F(\sqrt{26 \epsilon-37}) \text {, }
$$

dont le nombre de classes au sens restreint est 6. On trouve avec parfois 200 décimales de précision dans $\mathbb{C} / m_{F} \mathbb{Z}$ :

$$
\begin{aligned}
& \rho_{1}\left(\gamma_{1}\right) \approx 4.499999999999999 \ldots-0.728584512 \ldots i \\
& \rho_{2}\left(\gamma_{2}\right) \approx-1.078476376302846 \ldots-0.195385083050863 \ldots i \\
& \rho_{3}\left(\gamma_{3}\right) \approx-2.178476376302846 \ldots+0.195385083050863 \ldots i \\
& \rho_{4}\left(\gamma_{4}\right) \approx-18.61666666666666 \ldots+0.728584510266413 \ldots i \\
& \rho_{5}\left(\gamma_{5}\right) \approx-0.988190290363819 \ldots+0.195385083050863 \ldots i \\
& \rho_{6}\left(\gamma_{6}\right) \approx-2.421523623697153 \ldots-0.195385083050863 \ldots i
\end{aligned}
$$

La commande algdep $\left(u_{2}(1) e^{\frac{16}{15} i \pi}, 12\right)$ de Pari suggère la relation algébrique :

$$
\begin{array}{r}
Q_{2}(x)=x^{12}+106 x^{11}+873 x^{10}-2636 x^{9}+3040 x^{8}-626 x^{7}-1108 x^{6}-626 x^{5} \\
+3040 x^{4}+2636 x^{3}+873 x^{2}+106 x+1 .
\end{array}
$$

Ce polynôme est effectivement le polynôme minimal d'une unité de $H_{K}^{+}$. En outre, les nombres complexes

$$
u_{1}:=u_{1}(1)=-97.30316237461782 \ldots,
$$




$$
\begin{aligned}
& u_{2}:=u_{2}(1) e^{\frac{16}{15}} i \pi \approx-3.276785825745970 \ldots+0.955188763599790 \ldots i, \\
& u_{3}:=u_{3}(1) e^{\frac{19}{15} i \pi} \approx-0.281276149057161 \ldots+0.081992486337387 \ldots i, \\
& u_{4}:=u_{4}(1) e^{\frac{5}{15} i \pi} \approx-0.010277158271074 \ldots \\
& u_{5}:=u_{5}(1) e^{\frac{16}{15} i \pi} \approx-0.281276149057161 \ldots-0.081992486337387 \ldots i, \\
& u_{6}:=u_{6}(1) e^{\frac{16}{15} i \pi} \approx-3.276785825745970 \ldots-0.955188763599790 \ldots i
\end{aligned}
$$

coïncident chacun avec une racine de $Q_{2}$ sur plusieurs dizaines de décimales.

(c) Un exemple à groupe des classes $C_{2} \times C_{4}$. Le groupe des classes (au sens restreint) de $K=F(\sqrt{21 \epsilon-29})$ est d'ordre 8 , isomorphe à $C_{2} \times C_{4}$. L'algorithme décrit précédemment permet de calculer

$$
\begin{aligned}
& \rho_{1}\left(\gamma_{1}\right) \approx-1.866666666666 \ldots-0.787374943777 \ldots i, \\
& \rho_{2}\left(\gamma_{2}\right) \approx 0.297896510457 \ldots+0.068709821260 \ldots i, \\
& \rho_{3}\left(\gamma_{3}\right) \approx-0.300000000000 \ldots+0.161542382812 \ldots i, \\
& \rho_{4}\left(\gamma_{4}\right) \approx-1.097896510457 \ldots+0.068709821260 \ldots i, \\
& \rho_{5}\left(\gamma_{5}\right) \approx-0.133333333333 \ldots+0.787374943777 \ldots i, \\
& \rho_{6}\left(\gamma_{6}\right) \approx-0.031229843791 \ldots-0.068709821260 \ldots i, \\
& \rho_{7}\left(\gamma_{7}\right) \approx-0.900000000000 \ldots-0.161542382812 \ldots i, \\
& \rho_{8}\left(\gamma_{8}\right) \approx-1.38121 \ldots-0.391304 \ldots i .
\end{aligned}
$$

L'invariant le plus précis est $\rho_{5}\left(\gamma_{5}\right)$ dont on a obtenu 200 décimales significatives. La commande Pari algdep $\left(u_{5}(1) e^{-\frac{19}{15} i \pi}, 16,200\right)$ fournit comme candidat le polynôme réciproque

$$
\begin{aligned}
Q_{3}(x)=x^{16}+139 x^{15}-255 x^{14}-538 x^{13}+2018 x^{12}-2237 x^{11} \\
+1898 x^{10}-3034 x^{9}+4137 x^{8}-3034 x^{7}+1898 x^{6} \\
-2237 x^{5}+2018 x^{4}-538 x^{3}-255 x^{2}+139 x+1 .
\end{aligned}
$$

On constate d'abord que $Q_{3}$ est en effet le polynôme minimal d'une unité de $H_{K}^{+}$. Par ailleurs, six autres invariants coïncident eux aussi avec des racines de ce polynôme, au moins pour leurs $n$ premières décimales ( $10 \leq n \leq 200$ selon les cas) :

$$
\begin{aligned}
& u_{1}:=u_{1}(1) e^{\frac{16}{15} i \pi} \approx-140.7834195600 \ldots ; \\
& u_{2}:=u_{2}(1) e^{\frac{19}{15} i \pi} \approx 0.589707772431 \ldots-0.271949567981 \ldots i \\
& u_{3}:=u_{3}(1) e^{\frac{24}{15} i \pi} \approx-0.362402166665 \ldots ; \\
& u_{4}:=u_{4}(1) e^{\frac{5}{15} i \pi} \approx 0.589707772431 \ldots+0.271949567981 \ldots i ;
\end{aligned}
$$




$$
\begin{aligned}
& u_{5}:=u_{5}(1) e^{\frac{19}{15} i \pi} \approx-0.007103109180 \ldots ; \\
& u_{6}:=u_{6}(1) e^{\frac{3}{15} i \pi} \approx 1.398366700490 \ldots+0.644870625513 \ldots i ; \\
& u_{7}:=u_{7}(1) e^{\frac{12}{15} i \pi} \approx-2.759365401151 \ldots
\end{aligned}
$$

La précision avec laquelle $\rho_{8}\left(\gamma_{8}\right)$ est obtenu se révèle insuffisante pour identifier $u_{8}$ avec une des racines de $Q_{3}$. Pour des raisons de symétrie, il doit correspondre à la racine

$$
1.398366700490 \ldots-0.644870625513 \ldots i \text {. }
$$

6.2. Corps de base $\mathbb{Q}(\sqrt{2})$. L'anneau des entiers de $F^{\prime}=\mathbb{Q}(\sqrt{2})$ est euclidien pour la norme. On note $\epsilon=1+\sqrt{2}$ son unité fondamentale de norme -1 , et l'on ordonne les plongements de sorte que $\epsilon_{1}<0$ et $\epsilon_{2}>0$. La constante $m_{F^{\prime}}$ optimale est vraisemblablement $m_{F^{\prime}}=6$ dans ce cas.

(a) Un exemple à groupe des classes $C_{4}$. L'extension ATR $K=F^{\prime}(\sqrt{12 \epsilon-11})$ possède un groupe des classes au sens restreint cyclique d'ordre 4 . Nous associons à chaque classe un invariant dans $\mathbb{C} / m_{F^{\prime}} \mathbb{Z}$ :

$$
\begin{aligned}
& \rho_{1}\left(\gamma_{1}\right) \approx-1.333333333333333 \ldots-0.301378336840440 \ldots i \\
& \rho_{2}\left(\gamma_{2}\right) \approx-0.274078669810665 \ldots ; \\
& \rho_{3}\left(\gamma_{3}\right) \approx 0.166666666666666 \ldots+0.301378336840440 \ldots i \\
& \rho_{4}\left(\gamma_{4}\right) \approx-3.225921330189334 \ldots
\end{aligned}
$$

Tous sont obtenus avec une précision supérieure à 40 décimales. On en déduit au moyen de la commande Pari algdep le polynôme candidat

$$
Q_{4}(x)=x^{8}+6 x^{7}-5 x^{6}-4 x^{5}+5 x^{4}-4 x^{3}-5 x^{2}+6 x+1 .
$$

On vérifie a posteriori que ce polynôme définit bien une unité du corps de classes de Hilbert au sens restreint de $K$. Par ailleurs, 4 des 8 racines de $Q_{4}$ coïncident sur leurs 40 premières décimales avec les nombres complexes

$$
\begin{aligned}
& u_{1}:=u_{1}(1) e^{\frac{10}{6} i \pi} \approx-6.643347233735518 \ldots ; \\
& u_{2}:=u_{2}(1) e^{\frac{10}{6} i \pi} \approx-0.931490243381137 \ldots-0.363766307518644 \ldots i \\
& u_{3}:=u_{3}(1) e^{\frac{4}{6} i \pi} \approx-0.150526528994587 \ldots ; \\
& u_{4}:=u_{4}(1) e^{\frac{8}{6} i \pi} \approx-0.931490243381137 \ldots+0.363766307518644 \ldots i .
\end{aligned}
$$

(b) Un exemple à groupe des classes $C_{8}$. On considère enfin l'extension quadratique ATR $K=F^{\prime}(\sqrt{25 \epsilon-31})$, dont le groupe des classes au sens restreint est cyclique d'ordre 8 . À chaque classe correspond une matrice $\gamma_{k} \in \mathbf{S L}_{2}\left(\mathscr{O}_{F^{\prime}}\right)$ et un 
invariant de $\mathbb{C} / m_{F^{\prime}} \mathbb{Z}$ :

$$
\begin{aligned}
& \rho_{1}\left(\gamma_{1}\right) \approx-3.666666666666 \ldots-2.047636549497 \ldots i \\
& \rho_{2}\left(\gamma_{2}\right) \approx 1.855997078695 \ldots-0.315172999961 \ldots i \\
& \rho_{3}\left(\gamma_{3}\right) \approx-122.347 \ldots+0.625 \ldots i \\
& \rho_{4}\left(\gamma_{4}\right) \approx-87.06066958797 \ldots+0.315172999961 \ldots i \\
& \rho_{5}\left(\gamma_{5}\right) \approx-18.16666666666 \ldots+2.047636549497 \ldots i \\
& \rho_{6}\left(\gamma_{6}\right) \approx 20.060669587971 \ldots+0.315172999961 \ldots i \\
& \rho_{7}\left(\gamma_{7}\right) \approx-13.884816073095 \ldots ; \\
& \rho_{8}\left(\gamma_{8}\right) \approx 47.894002921304 \ldots-0.3151729999612 \ldots i
\end{aligned}
$$

L'invariant le plus précis est $\rho_{5}\left(\gamma_{5}\right)$, qu'on a pu calculer avec plus de 200 décimales significatives. La commande Pari algdep $\left(u_{5}(1) e^{-\frac{8}{16} i \pi}, 16\right)$ suggère le polynôme réciproque

$$
\begin{aligned}
Q_{5}(x):=x^{16}+ & 386792 x^{15}-5613916 x^{14}+21963312 x^{13} \\
& -13291318 x^{12}+32052888 x^{11}+15011472 x^{10}+16774296 x^{9} \\
& +36336275 x^{8}+16774296 x^{7}+15011472 x^{6}+32052888 x^{5} \\
& -13291318 x^{4}+21963312 x^{3}-5613916 x^{2}+386792 x+1 .
\end{aligned}
$$

Il est aisé de vérifier que $Q_{5}$ définit effectivement une unité de $H_{K}^{+}$. À une racine de l'unité près, les exponentielles des nombres complexes précédents coïncident sur leurs premières décimales (entre 10 et 200 selon les cas) avec les racines suivantes de $Q_{5}$ :

$$
\begin{aligned}
& u_{1}:=u_{1}(1) e^{\frac{2}{6} i \pi} \approx-386806.513645927 \ldots ; \\
& u_{2}:=u_{2}(1) e^{\frac{2}{6} i \pi} \approx 7.17151519909699 \ldots+1.02818667270890 \ldots i \\
& u_{4}:=u_{4}(1) e^{\frac{1}{6} i \pi} \approx 0.136632045175690 \ldots+0.0195890608908274 \ldots i \\
& u_{5}:=u_{5}(1) e^{\frac{8}{6} i \pi} \approx-0.00000258527187 \ldots ; \\
& u_{6}:=u_{6}(1) e^{\frac{11}{6} i \pi} \approx 0.136632045175690 \ldots-0.0195890608908274 \ldots i \\
& u_{7}:=u_{7}(1) e^{\frac{7}{6} i \pi} \approx-0.317863811003618 \ldots-0.948136381357796 \ldots i \\
& u_{8}:=u_{8}(1) e^{\frac{1}{6} i \pi} \approx 7.17151519909699 \ldots-1.02818667270890 \ldots i .
\end{aligned}
$$

La précision obtenue sur les décimales de $\rho_{3}\left(\gamma_{3}\right)$ est insuffisante pour identifier $u_{3}$. Pour des raisons de symétrie, il doit correspondre à la racine suivante de $Q_{5}$ : 


\section{Périodes de séries d'Eisenstein.}

L'objet de cette partie est d'établir une formule générale qui exprime la valeur spéciale en $s=0$ des fonctions $L$ introduites précédemment en termes de périodes de séries d'Eisenstein pour un tore de $\Gamma$, ce qui complète la démonstration des théorèmes 2.1 et 3.2 .

Des formules similaires ont déjà été obtenues dans [Haran 1987] et [Hara 1993] par exemple. On donne ici une présentation des résultats exposés dans la section 5 de la thèse [Charollois 2004] sous une forme directement utilisable dans les sections 2 et 3.

Quelques notations multi-indices standard permettront de rendre les formules plus agréables. On associe d'abord à un $n$-uplet $z=\left(z_{1}, \ldots, z_{n}\right)$ de nombres complexes sa partie imaginaire $y=\left(\operatorname{Im} z_{1}, \ldots, \operatorname{Im} z_{n}\right)$, sa trace $\operatorname{Tr}(z)=z_{1}+\cdots+z_{n}$ et sa norme $N(z)=z_{1} \cdots z_{n}$. Pour un élément $\mu$ de $F$, on désigne par $\mu z$ et $z+\mu$ les $n$-uplets $\left(\mu_{1} z_{1}, \ldots, \mu_{n} z_{n}\right)$ et $\left(\mu_{1}+z_{1}, \ldots, \mu_{n}+z_{n}\right)$ respectivement. On introduit alors pour $\operatorname{Re} s>1$ la série d'Eisenstein

$$
E(z, s)=\sum_{(\mu, v) \in \mathscr{O}_{F}^{2} / \mathcal{O}_{F}^{\times}}^{\prime} \frac{N(y)^{s}}{|N(\mu z+v)|^{2 s}},
$$

où le groupe d'unités $\mathcal{O}_{F}^{\times}$opère diagonalement sur $\mathscr{O}_{F}^{2}$. Cette série définit une forme modulaire de Hilbert non-holomorphe de poids $(0, \ldots, 0)$ pour $\Gamma$. Le théorème principal de cette partie met en jeu des périodes associées à des dérivées partielles de $E(z, s)$.

Soit $K$ une extension quadratique de $F$. La signature de $K$ est de la forme $(2 r, c)$ avec $r+c=n$. On ordonne les $n$ places archimédiennes de $F$ de sorte que les $c$ premières places $v_{1}, \ldots, v_{c}$ se prolongent chacune en une place complexe de $K$, et que les $r$ places suivantes $v_{c+1}, \ldots, v_{n}$ donnent lieu à un isomorphisme de $\mathbb{R}$-algèbres $K \otimes_{F, v_{j}} \mathbb{R} \simeq \mathbb{R} \oplus \mathbb{R}$. On fixe une fois pour toutes de telles identifications, que l'on appelle encore $v_{j}$ par abus de notation.

Comme dans la section 1.2, on fixe un idéal $I$ de $\mathscr{O}_{F}$, et l'on note $\mathscr{O}_{I}=\mathscr{O}_{F}+I \mathscr{O}_{K}$ l'ordre de $K$ de conducteur $I$. On se donne un $O_{I}$-module projectif $M$ de $K$, et l'on définit $\tau:=\omega_{2} / \omega_{1}$, où $\left(\omega_{1}, \omega_{2}\right)$ est une une $\mathcal{O}_{F}$-base positive de $M$. On pose alors

$$
\begin{aligned}
& \tau_{j}:=v_{j}(\tau) \in \mathscr{H}_{j} \quad \text { pour } j=1, \ldots, c, \\
& \left(\tau_{j}, \tau_{j}^{\prime}\right):=v_{j}(\tau) \in \mathbb{R} \times \mathbb{R} \quad \text { pour } j=c+1, \ldots, n .
\end{aligned}
$$

Pour chaque $c+1 \leq j \leq n$, on appelle $\Upsilon_{j}$ la géodésique hyperbolique sur $\mathscr{H}_{j}$ joignant $\tau_{j}$ à $\tau_{j}^{\prime}$, orientée dans le sens allant de $\tau_{j}^{\prime}$ à $\tau_{j}$.

Le produit

$$
R_{\tau}=\left\{\tau_{1}\right\} \times \cdots\left\{\tau_{c}\right\} \times \Upsilon_{c+1} \times \cdots \times \Upsilon_{n} \subset \mathscr{H}^{n}
$$


est un espace contractile homéomorphe à $\mathbb{R}^{r}$. On le munit de l'orientation naturelle héritée des $\Upsilon_{j}$. Le stabilisateur $\Gamma_{\tau}$ de $\tau$ dans $\Gamma$ est un groupe abélien de rang $r$ (modulo la torsion), qui s'identifie avec le sous-groupe $V_{1}$ des unités de $V$ de norme relative 1 sur $F$. Il opère sur $R_{\tau}$ par homographies, et le quotient $\Gamma_{\tau} \backslash R_{\tau}$ est compact, isomorphe à un tore réel de dimension $r$. Soit $\Delta_{\tau}$ un domaine fondamental pour l'action de $\Gamma_{\tau}$ sur $R_{\tau}$. On identifie $\Delta_{\tau}$ avec son image dans $X$, qui est un cycle fermé de dimension $r$ dans ce quotient.

Théorème 7.1. Pour tout $O_{I}$-module projectif $M$ dans $K$, on $a$ :

$$
\int_{\Delta_{\tau}} \frac{\partial^{r} E(z, s)}{\partial z_{c+1} \cdots \partial z_{n}} d z_{c+1} \wedge \cdots \wedge d z_{n}=\left(\frac{\Gamma\left(\frac{s+1}{2}\right)^{2}}{2 i \Gamma(s)}\right)^{r} d_{F}^{-s} L(M, s) .
$$

Démonstration. On associe à tout nombre complexe $s$ la $r$-forme différentielle $\Gamma$ invariante sur $\mathscr{H}^{n}$

$$
\omega_{\text {Eis }}^{r}(s):=\frac{\partial^{r} E(z, s)}{\partial z_{c+1} \cdots \partial z_{n}} d z_{c+1} \wedge \cdots \wedge d z_{n} .
$$

Lorsque $\operatorname{Re} s>1$, un calcul direct montre que la période considérée prend la forme

$$
\begin{aligned}
\int_{\Delta_{\tau}} \omega_{\text {Eis }}^{r}(s)=\left(\frac{s}{2 i}\right)^{r} \int_{\Delta_{\tau}} \sum_{(\mu, v) \in \mathbb{O}_{F}^{2} / \mathbb{O}_{F}^{\times}}^{\prime}( & \left.\prod_{j=1}^{c} \frac{\left(\operatorname{Im} \tau_{j}\right)^{s}}{\left|\mu_{j} \tau_{j}+v_{j}\right|^{2 s}}\right) \\
& \times\left(\bigwedge_{j=c+1}^{n} \frac{y_{j}^{s-1}\left(\mu_{j} \bar{z}_{j}+v_{j}\right)^{2}}{\left|\mu_{j} z_{j}+v_{j}\right|^{2 s+2}} d z_{j}\right) .
\end{aligned}
$$

On observe d'abord que

$$
N(M)=d_{F} \prod_{j=1}^{c} \operatorname{Im} \tau_{j} \prod_{j=c+1}^{n}\left(\tau_{j}^{\prime}-\tau_{j}\right) .
$$

On définit une action naturelle de $K^{\times}$(et donc du groupe $V_{1}$ ) sur $\left(\mathbb{R}_{+}^{\times}\right)^{r}$ par la formule

$$
\alpha \cdot\left(t_{c+1}, \ldots, t_{n}\right):=\left(\left|\frac{\alpha_{c+1}}{\alpha_{c+1}^{\prime}}\right| t_{c+1}, \ldots,\left|\frac{\alpha_{n}}{\alpha_{n}^{\prime}}\right| t_{n}\right) .
$$

Le tore compact réel $T^{r}: V_{1} \backslash\left(\mathbb{R}_{+}^{\times}\right)^{r}$ est muni d'une mesure de Haar canonique

$$
d^{\times} t=\frac{d t_{c+1}}{t_{c+1}} \wedge \cdots \wedge \frac{d t_{n}}{t_{n}} .
$$

On peut supposer que $(1, \tau)$ est une $\hat{O}_{F}$-base positive de $M$, quitte à changer $M$ en $\alpha M$ avec $\alpha \in K^{\times}$. Dans ce cas, la géodésique $\Upsilon_{j}$ est orientée dans le sens trigonométrique selon nos conventions. En posant $t_{j}=-i\left(z_{j}-\tau_{j}^{\prime}\right) /\left(z_{j}-\tau_{j}\right)$ on 
obtient une paramétrisation $t_{j} \in \mathbb{R}_{+}^{\times}$de cette géodésique qui permet d'identifier le quotient $\Gamma_{\tau} \backslash R_{\tau}$ avec le tore $T^{r}$ en respectant les orientations.

Le changement de variable qui correspond à cette paramétrisation transforme l'identité (41) en l'expression

$$
\int_{\Delta_{\tau}} \omega_{\text {Eis }}^{r}(s)=\left(\frac{s}{2}\right)^{r}\left(\frac{N(M)}{d_{F}}\right)^{s} \int_{T^{r}} \sum_{\beta \in M / \mathbb{O}_{F}^{\times}}^{\prime}\left|N_{K / \mathbb{Q}}(\beta)\right|^{-s} g_{\beta}(\beta \cdot t) d^{\times} t,
$$

où l'on a posé $\beta=\mu \tau+v$, élément de $K^{\times}$qui parcourt les classes non-nulles de $M / \mathscr{O}_{F}^{\times}$quand le couple $(\mu, v)$ parcourt les classes non-nulles de $\mathscr{O}_{F}^{2} / \mathscr{O}_{F}^{\times}$, et où $g_{\beta}:\left(\mathbb{R}_{+}^{\times}\right)^{r} \longrightarrow \mathbb{C}$ désigne la fonction auxiliaire

$$
g_{\beta}(t)=\prod_{j=c+1}^{n} \frac{t_{j}^{s}\left(-i t_{j}+\operatorname{signe}\left(\beta_{j} \beta_{j}^{\prime}\right)\right)^{2}}{\left(t_{j}^{2}+1\right)^{s+1}} .
$$

L'étape cruciale consiste maintenant à utiliser une idée due à Hecke [Siegel 1980, p. 86] : on observe d'abord que l'on obtient un système de représentants des classes non-nulles de $M / O_{F}^{\times}$en considérant la famille $\{\beta \epsilon\}$, lorsque $\beta$ parcourt les classes non-nulles de $M / \tilde{V}$ et $\epsilon$ parcourt $V_{1} /\{ \pm 1\}$.

Par conséquent, l'identité (42) devient

$$
\begin{aligned}
\int_{\Delta_{\tau}} \omega_{\text {Eis }}^{r}(s) & \\
= & \left(\frac{s}{2}\right)^{r}\left(\frac{N(M)}{d_{F}}\right)^{s} \sum_{\beta \in M / \tilde{V}}^{\prime}\left|N_{K / \mathbb{Q}}(\beta)\right|^{-s} \int_{V_{1} \backslash\left(\mathbb{R}_{+}^{\times}\right)^{r}} \sum_{\epsilon \in V_{1} /\{ \pm 1\}} g_{\beta \epsilon}(\beta \epsilon \cdot t) d^{\times} t \\
= & \left(\frac{s}{2}\right)^{r}\left(\frac{N(M)}{d_{F}}\right)^{s} \sum_{\beta \in M / \tilde{V}}^{\prime}\left|N_{K / \mathbb{Q}}(\beta)\right|^{-s} \int_{\left(\mathbb{R}_{+}^{\times}\right)^{r}} g_{\beta}(\beta \cdot t) d^{\times} t
\end{aligned}
$$

Le changement de variable $u=\beta \cdot t$ dans la dernière intégrale permet de scinder cette intégrale multiple en un produit de $r$ intégrales de la forme suivante [Siegel 1980, formule (107)] :

$$
\int_{0}^{+\infty} \frac{u_{j}^{s}\left(-i u_{j}+\operatorname{signe}\left(\beta_{j} \beta_{j}^{\prime}\right)\right)^{2}}{\left(u_{j}^{2}+1\right)^{s+1}} \frac{d u_{j}}{u_{j}}=-i \operatorname{signe}\left(\beta_{j} \beta_{j}^{\prime}\right) \frac{\Gamma\left(\frac{s+1}{2}\right)^{2}}{\Gamma(s+1)} .
$$

Il s'ensuit que

$$
\begin{aligned}
& \int_{\Delta_{\tau}} \omega_{\text {Eis }}^{r}(s) \\
& \quad=\left(\frac{s \Gamma\left(\frac{s+1}{2}\right)^{2}}{2 i \Gamma(s+1)}\right)^{r}\left(\frac{N(M)}{d_{F}}\right)^{s} \sum_{\beta \in M / \tilde{V}}^{\prime}\left|N_{K / \mathbb{Q}}(\beta)\right|^{-s} \prod_{j=c+1}^{n} \operatorname{signe}\left(\beta_{j} \beta_{j}^{\prime}\right) .
\end{aligned}
$$


La formule (40) s'en déduit immédiatement au vu de la définition (21) de $L(M, s)$.

Corollaire 7.2. Soit $K$ une extension quadratique de signature $(2 r, c) d u$ corps $F$, avec $r+c=n=[F: \mathbb{Q}]$. Soit $M$ un $\mathbb{O}_{I}$-module projectif dans $K$. La fonction $L(M, s)$ possède alors un zéro d'ordre $\geq c$ en $s=0$, et l'on a les formules :

i. si $r \geq 2$, alors:

$$
\frac{L^{(c)}(M, 0)}{c !}=\frac{(2 i)^{r}}{2^{n} \pi^{r}} \int_{\Delta_{\tau}} \frac{\partial^{r} \tilde{h}(z)}{\partial z_{c+1} \ldots \partial z_{n}} d z_{c+1} \wedge \cdots \wedge d z_{n} .
$$

ii. si le corps $K$ n'a que deux places réelles $(r=1)$, alors

$$
\frac{L^{(n-1)}(M, 0)}{(n-1) !}=\frac{2 i}{2^{n} \pi} \int_{\Delta_{\tau}}\left(\frac{\partial \tilde{h}(z)}{\partial z_{n}}-\frac{2^{2 n-2} R_{F}}{z_{n}-\bar{z}_{n}}\right) d z_{n} .
$$

Démonstration. D'après [Asai 1970, théorème 3] (voir aussi le théorème 2.1 de [Charollois 2007]), la fonction $E(z, s)$ se prolonge sur $\mathbb{C}$ en une fonction méromorphe de la variable $s$ qui satisfait l'équation fonctionnelle

$$
G(2 s) E(z, s)=G(2-2 s) E(z, 1-s)
$$

avec $G(s):=d_{F}^{s / 2} \pi^{-n s / 2} \Gamma(s / 2)^{n}$. En outre, elle possède un unique pôle simple en $s=1$, et les premiers termes de son développement de Laurent au voisinage de ce pôle sont fournis par la formule limite de Kronecker généralisée :

$$
E(z, s)=\frac{(2 \pi)^{n} R_{F}}{4 d_{F}}\left(\frac{1}{s-1}+\gamma_{F}-\log \prod_{j=1}^{n} y_{j}+h(z)\right)+O(s-1),
$$

où $\gamma_{F}$ est une constante qui ne dépend que de $F$, et où les deux fonctions $h$ et $\tilde{h}=4^{n-1} R_{F} h$ ont été introduites en (9) et (10). Les deux égalités précédentes permettent d'obtenir le développement de Taylor de $E(z, s)$ au voisinage de $s=0$ :

$$
E(z, s)=-2^{n-2} R_{F} s^{n-1}-2^{n-2} R_{F} s^{n}\left(\log N(y)+\gamma_{F}^{\prime}-h(z)\right)+O\left(s^{n+1}\right),
$$

où $\gamma_{F}^{\prime}$ ne dépend que de $F$. On trouve par conséquent pour $r=n-c \geq 2$ :

$$
\frac{\partial^{r} E(z, s)}{\partial z_{c+1} \ldots \partial z_{n}}=\frac{s^{n}}{2^{n}} \frac{\partial^{r} \tilde{h}(z)}{\partial z_{c+1} \ldots \partial z_{n}}+O\left(s^{n+1}\right) .
$$

Pour $r=1$, on a un terme supplémentaire :

$$
\frac{\partial E(z, s)}{\partial z_{n}}=\frac{s^{n}}{2^{n}}\left(\frac{\partial \tilde{h}(z)}{\partial z_{n}}-\frac{2^{2 n-2} R_{F}}{z_{n}-\bar{z}_{n}}\right)+O\left(s^{n+1}\right) .
$$


On conclut alors du théorème 7.1 que $L(M, s)$ se prolonge en une fonction méromorphe sur $\mathbb{C}$, dont le développement de Taylor au voisinage de $s=0$ se déduit des identités (50) et (51) ci-dessus :

$$
L(M, s)=\frac{(2 i)^{r} s^{n-r}}{2^{n} \pi^{r}} \int_{\Delta_{\tau}} \frac{\partial^{r} \tilde{h}(z)}{\partial z_{c+1} \ldots \partial z_{n}} d z_{c+1} \wedge \cdots \wedge d z_{n}+O\left(s^{n+1-r}\right)
$$

pour $r \geq 2$, tandis que pour $r=1$ :

$$
L(M, s)=\frac{2 i s^{n-1}}{2^{n} \pi} \int_{\Delta_{\tau}}\left(\frac{\partial \tilde{h}(z)}{\partial z_{n}}-\frac{2^{2 n-2} R_{F}}{z_{n}-\bar{z}_{n}}\right)+O\left(s^{n}\right) .
$$

Les formules (45) et (46) souhaitées en résultent immédiatement.

\section{Remerciements}

Le premier auteur tient à remercier chaleureusement Philippe Cassou-Noguès et Martin Taylor qui sont à l'origine de son travail de thèse. Les deux auteurs ont aussi bénéficié de nombreux échanges avec Samit Dasgupta au sujet du présent article.

Charollois a bénéficié du soutien matériel de l'Institut de Mathématiques de Bordeaux et du CRM-ISM de Montréal au cours de l'élaboration de cet article. Le travail de Darmon a été financé en partie par le CRSNG et la chaire James McGill.

\section{Références}

[Asai 1970] T. Asai, “On a certain function analogous to $\log |\eta(z)|$ ”, Nagoya Math. J. 40 (1970), 193-211. MR 42 \#5921 Zbl 0213.05701

[Charollois 2004] P. Charollois, Sommes de Dedekind et périodes de formes modulaires de Hilbert, Thèse de doctorat, Université Bordeaux I, 2004.

[Charollois 2007] P. Charollois, "Sommes de Dedekind associées à un corps de nombres totalement réel”, J. Reine Angew. Math. 610 (2007), 125-147. MR 2359852

[Cooke 1976] G. E. Cooke, "A weakening of the Euclidean property for integral domains and applications to algebraic number theory, I", J. Reine Angew. Math. 282 (1976), 133-156. MR 53 \#10758a Zbl 0328.13013

[Darmon 2001] H. Darmon, "Integration on $\mathscr{H}_{p} \times \mathscr{H}_{\text {and }}$ arithmetic applications", Ann. of Math. (2) 154 :3 (2001), 589-639. MR 2003j :11067 Zbl 1035.11027

[Darmon 2004] H. Darmon, Rational points on modular elliptic curves, CBMS Regional Conference Series in Mathematics 101, Published for the Conference Board of the Mathematical Sciences, Washington, DC, 2004. MR 2004k :11103 Zbl 1057.11034

[Darmon et Dasgupta 2006] H. Darmon et S. Dasgupta, "Elliptic units for real quadratic fields", Ann. of Math. (2) 163 :1 (2006), 301-346. MR 2007a :11079 Zbl 1130.11030

[Darmon et Logan 2003] H. Darmon et A. Logan, "Periods of Hilbert modular forms and rational points on elliptic curves", Int. Math. Res. Not. 2003 :40 (2003), 2153-2180. MR 2005f :11110 Zbl 1038.11035 
[Dasgupta 1999] S. Dasgupta, Stark's conjectures, senior thesis, Harvard University, Cambridge, MA, 1999.

[Dummit et al. 2004] D. S. Dummit, B. A. Tangedal et P. B. van Wamelen, "Stark's conjecture over complex cubic number fields", Math. Comp. 73 :247 (2004), 1525-1546. MR 2005b :11181 Zbl 1046.11082

[Freitag 1990] E. Freitag, Hilbert modular forms, Springer, Berlin, 1990. MR 91c :11025 Zbl 0702. 11029

[van der Geer 1988] G. van der Geer, Hilbert modular surfaces, Ergebnisse der Mathematik (3) 16, Springer, Berlin, 1988. MR 89c :11073 Zbl 0634.14022

[Goren et Lauter 2007] E. Z. Goren et K. E. Lauter, "Class invariants for quartic CM fields", Ann. Inst. Fourier (Grenoble) 57 :2 (2007), 457-480. MR 2310947 Zbl 05176593

[Greenberg 2008] M. Greenberg, "Stark-Heegner points and the cohomology of quaternionic Shimura varieties", prépublication, 2008, Voir math.ucalgary.ca/ mgreenbe/shp.pdf.

[Hara 1993] Y. Hara, "On calculation of $L_{K}(1, \chi)$ for some Hecke characters", J. Math. Kyoto Univ. 33 :3 (1993), 865-898. MR 94j :11114 Zbl 0795.11061

[Haran 1987] S. Haran, “p-adic L-functions for modular forms”, Compositio Math. 62 :1 (1987), 31-46. MR 88k :11036 Zbl 0618.10027

[Harder 1975] G. Harder, "On the cohomology of $S L(2, O)$ ", pp. 139-150 dans Lie groups and their representations (Budapest, 1971), Halsted, New York, 1975. MR 54 \#12977 Zbl 0395.57028

[Kirby et Melvin 1994] R. Kirby et P. Melvin, "Dedekind sums, $\mu$-invariants and the signature cocycle”, Math. Ann. 299 :2 (1994), 231-267. MR 95h :11042 Zbl 0809.11027

[Ren et Sczech 2008] T. Ren et R. Sczech, “A refinement of Stark's conjecture over complex cubic number fields", prépublication, 2008.

[de Shalit et Goren 1997] E. de Shalit et E. Z. Goren, "On special values of theta functions of genus two", Ann. Inst. Fourier (Grenoble) 47 :3 (1997), 775-799. MR 98g :11071 Zbl 0909.15001

[Siegel 1980] C. L. Siegel, Advanced analytic number theory, 2nd éd., Studies in Mathematics 9 , Tata Institute of Fundamental Research, Bombay, 1980. MR 83m :10001 Zbl 0478.10001

[Stark 1976] H. M. Stark, " $L$-functions at $s=1$, III : Totally real fields and Hilbert's twelfth problem”, Advances in Math. 22 :1 (1976), 64-84. MR 55 \#10427 Zbl 0348.12017

[Tate 1984] J. T. Tate, Les conjectures de Stark sur les fonctions L d'Artin en $s=0$, Progress in Math. 47, Birkhäuser, Boston, 1984. MR 86e :11112 Zbl 0545.12009

[Trifković 2006] M. Trifković, "Stark-Heegner points on elliptic curves defined over imaginary quadratic fields", Duke Math. J. 135 :3 (2006), 415-453. MR 2008d :11064 Zbl 1111.14025

Communicated by Bjorn Poonen

Received 2008-05-21 Accepted 2008-06-25

charollois@math.jussieu.fr_Institut de mathématiques de Jussieu,

Équipe de théorie des nombres, Case 247-4, place Jussieu, 75252 Paris Cedex, France

http://www.math.jussieu.fr/ charollois/pageperso.html

darmon@math.mcgill.ca The Department of Mathematics and Statistics,

McGill University, 805 Sherbrooke Street West,

Montreal, QC H3A2K6, Canada

http://www.math.mcgill.ca/darmon/ 